\title{
Eigenvalue analysis and convergence acceleration techniques for summation-by-parts approximations
}

Andrea Alessandro Ruggiu 
Linköping Studies in Science and Technology.

Dissertations, No. 2002

\title{
Eigenvalue analysis and convergence acceleration techniques for summation-by-parts approximations
}

\author{
Andrea Alessandro Ruggiu
}

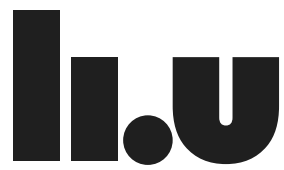

LINKÖPING UNIVERSITY

Department of Mathematics, Division of Computational Mathematics Linköping University, SE-581 83 Linköping, Sweden

Linköping 2019 
This work was supported by VINNOVA, the Swedish Governmental Agency for Innovation Systems, under contract number 2013-01209.

Linköping Studies in Science and Technology. Dissertations, No. 2002

Eigenvalue analysis and convergence acceleration techniques for summationby-parts approximations

Copyright (C) Andrea Alessandro Ruggiu, 2019

Division of Computational Mathematics

Department of Mathematics

Linköping University

SE-581 83, Linköping, Sweden

andrea.ruggiu@liu.se

www.liu.se/mai

Typeset by the author in $\mathrm{IAT}_{\mathrm{E}} \mathrm{X} 2 \mathrm{e}$ documentation system.

ISSN 0345-7524

ISBN 978-91-7685-023-7

Printed by LiU-Tryck, Linköping, Sweden 2019 
To my mother. 

Animum debes mutare non caelum. (You should change your state of mind, not the sky)

Lucius Annaeus Seneca 



\section{Abstract}

Many physical phenomena can be described mathematically by means of partial differential equations. These mathematical formulations are said to be wellposed if a unique solution, bounded by the given data, exists. The boundedness of the solution can be established through the so-called energy-method, which leads to an estimate of the solution by means of integration-by-parts. Numerical approximations mimicking integration-by-parts discretely are said to fulfill the Summation-By-Parts (SBP) property. These formulations naturally yield bounded approximate solutions if the boundary conditions are weakly imposed through Simultaneous-Approximation-Terms (SAT). Discrete problems with bounded solutions are said to be energy-stable.

Energy-stable and high-order accurate SBP-SAT discretizations for well-posed linear problems were first introduced for centered finite-difference methods. These mathematical formulations, based on boundary conforming grids, allow for an exact mimicking of integration-by-parts. However, other discretizations techniques that do not include one or both boundary nodes, such as pseudospectral collocation methods, only fulfill a generalized SBP (GSBP) property but still lead to energy-stable solutions.

This thesis consists of two main topics. The first part, which is mostly devoted to theoretical investigations, treats discretizations based on SBP and GSBP operators. A numerical approximation of a conservation law is said to be conservative if the approximate solution mimics the physical conservation property. It is shown that conservative and energy-stable spatial discretizations of variable coefficient problems require an exact numerical mimicking of integration-by-parts. We also discuss the invertibility of the algebraic problems arising from $(\mathrm{G}) \mathrm{SBP}$ SAT discretizations in time of energy-stable spatial approximations. We prove that pseudo-spectral collocation methods for the time derivative lead to invertible fully-discrete problems. The same result is proved for second-, fourth- and sixth-order accurate finite-difference based time integration methods.

Once the invertibility of (G)SBP-SAT discrete formulations is established, we are interested in efficient algorithms for the unique solution of such problems. To this end, the second part of the thesis has a stronger experimental flavour and deals with convergence acceleration techniques for SBP-SAT approximations. First, we consider a modified Dual Time-Stepping (DTS) technique which makes use of two derivatives in pseudo-time. The new DTS formulation, compared to the classical one, accelerates the convergence to steady-state and reduces the stiffness of the problem. Next, we investigate multi-grid methods. For 
parabolic problems, highly oscillating error modes are optimally damped by iterative methods, while smooth residuals are transferred to coarser grids. In this case, we show that the Galerkin condition in combination with the SBPpreserving interpolation operators leads to fast convergence. For hyperbolic problems, low frequency error modes are rapidly expelled by grid coarsening, since coarser grids have milder stability restrictions on time steps. For such problems, Total Variation Dimishing Multi-Grid (TVD-MG) allows for faster wave propagation of first order upwind discretizations. In this thesis, we extend low order TVD-MG schemes to high-order SBP-SAT upwind discretizations. 


\section{Sammanfattning på svenska}

Fysikaliska fenomen och kemiska processer kan beskrivas med partiella differentialekvationer. Dessa ekvationer kan nästan aldrig lösas analytiskt, utan istället formulerar man en diskret motsvarighet till det kontinuerliga problemet som löses med en numerisk metod. Man får då en approximation till den riktiga lösningen. Det diskreta problemet kan formuleras på olika sätt och varje enskilt sätt innebär någon form av informationsförlust. I korthet är beräkningsmatematik den gren inom matematiken som behandlar just översättningen från det kontinuerliga till det diskreta problemet.

Den här avhandlingen behandlar olika numeriska approximationer som uppfyller den diskreta motsvarigheten till partiell integration, vilket är en regel som används för att bevisa välställdhet hos differentialekvationer med begynnelseoch randvillkor. Dessa approximationer behåller viktiga egenskaper som det ursprungliga kontinuerliga problemet har såsom stabilitet, konservation och inverterbarhet. Den första delen av avhandlingen behandlar just dessa egenskaper hos den diskreta formuleringen. Först visar vi att diskretiseringar av problem med variabla koefficienter kräver randnoder för att förbli både stabila och konservativa. Sedan bevisas inverterbarhet hos olika tidsstegningsmetoder.

Det andra huvudsakliga bidraget i avhandlingen handlar om hur dessa inverterbara problem löses effektivt. En modifierad dual tidsstegning med andraderivatan undersöks, samt två nya typer av multigridmetoder. Till att börja med jämför vi den vanliga duala tidsstegningen med den nya och visar att den leder till snabbare konvergens. Dessutom utvecklar vi de nya multigridmetoderna så att övergångsfasen till det tidsoberoende tillståndet blir snabbare. Den första multigridmetoden, som används till elliptiska problem, inkluderar nya interpolationsoperatorer som är konsistenta med partiell integration. Den andra multigridmetoden ökar våghastigheten när den används på hyperboliska problem. 



\section{List of Papers}

This thesis is based on the following papers, which will be referred to by their roman numerals:

I. J. Nordström, A. A. Ruggiu, On conservation and stability properties for summation-by-parts schemes, Journal of Computational Physics, Vol. 344, pp. 451-464, 2017.

II. A. A. Ruggiu, J. Nordström, On pseudo-spectral time discretizations in summation-by-parts form, Journal of Computational Physics, Vol. 360, pp. 192-201, 2018.

III. A. A. Ruggiu, J. Nordström, Eigenvalue analysis for summation-by-parts finite difference time discretizations. LiTH-MAT-R-2019/09-SE. Submitted for publication (2019).

IV. J. Nordström, A. A. Ruggiu, Dual time-stepping using second derivatives, Journal of Scientific Computing, https://doi.org/10.1007/s10915019-01047-5, pp. 1-22, 2019.

V. A. A. Ruggiu, P. Weinerfelt, J. Nordström, A new multigrid formulation for high order finite difference methods on summation-by-parts form, Journal of Computational Physics, Vol. 359, pp. 216-238, 2018.

VI. A. A. Ruggiu, J. Nordström, Multigrid schemes for high order discretizations of hyperbolic problems, Conference proceedings of AIAA Scitech 2019 Forum, San Diego, California. AIAA 2019-0103, 2019.

I have written the manuscripts myself with editorial support from the coauthors, Jan Nordström (papers I-VI) and Per Weinerfelt (paper V). I have derived most of the novel theoretical findings and conducted all numerical experiments. 



\section{Acknowledgements}

Professor Jan Nordström deserves to be acknowledged first. He has been the person who has believed in me the most since we met the first time, giving me the chance to challenge myself with difficult and stimulating research tasks. Above all, I wish to thank him and Nils-Hassan Quttineh for giving me the opportunity to be the lecturer of the course Beräkningsmatematik, last year. It has been undoubtedly one of the best experiences in my life. Next, I would like to thank my co-supervisor, Professor Per Weinefelt, for his support and encouragement, especially in the first stages of my doctoral studies.

I am also grateful to all the fellow Ph.D. students at the mathematics department with whom I have shared all or part of my journey. Among them, I would like to thank in particular Markus and Fatemeh for having made my working hours great and Oskar, Fredrik, Roghi and Nancy for the beautiful memories, both in and outside LiU. A special and huge thanks goes to Cristina, without whom I would have neither been here nor defended this thesis. It would be too difficult to express in words my gratitude to her.

In these five years I have been lucky enough to get in touch with wonderful people here in Linköping even outside the university. Without them, my experience in this city would have been less valuable. First of all, Irene with whom I shared $n$ evenings talking, eating and drinking. I am also glad to have met Isabella, to whom I am grateful for all the nice fikas and chats. Probably, I would have not survived here without Sara and Marco: thanks for having opened Le Borgate and for all the wonderful evenings I have spent there. Thanks also to Abramo for being such a good friend and Giovanni for hitting regularly the gym(s) with me. Last, but not least, I want to especially acknowledge Melinda for having given me the strength to write this thesis. She has been able to bring out the best in me during the last months of this experience.

I wish to thank also all my friends not living in Sweden. In particular, Paolo with whom I traveled across Europe in these five years and Emma who offered me her constant support when it was needed the most. Titus for all the milanese afternoons we spent together and my former university colleagues and friends Alessandro and Sofia for our "work" lunches, when I had been in town. Alessandro, Andrea, Carlo and Michele for all the exceptional (Ruggiu) stories we have shared these years. Andrea and Camilla, as well as Carla and her wonderful family, for having provided inexhaustible food and drink supplies to heal my homesickness. Raul, Miguel, Jonathan and Cristhian for having raised my alcohol tolerance through extensive drinking sessions at the pub. 
Finally, I want to acknowledge my family who has always been by my side during this adventure: my brother, my dad and Angela. Thanks for all your unconditional support.

Andrea Alessandro Ruggiu

Linköping, 2019 


\section{Contents}

Abstract $\quad$ v

Sammanfattning på svenska vii

List of Papers $\quad$ ix

Acknowledgements $\quad x i$

1 Introduction $\quad 1$

2 Conservation, energy boundedness and well-posedness 3

3 Summation-By-Parts operators 5

3.1 Finite-difference approximations .............. 5

3.1.1 Centered finite-differences . . . . . . . . . . . . 5

3.1.2 Upwind difference operators . . . . . . . . . . . . . 7

3.2 Pseudo-spectral collocation methods . . . . . . . . . . 8

3.3 Multi-dimensional SBP operators . . . . . . . . . . . . . 9

4 Conservation and stability for SBP-SAT approximations $\quad 11$

4.1 The splitting technique . . . . . . . . . . . . . 11

4.2 GSBP-SAT approximations . . . . . . . . . . . . . . 12

5 The eigenvalues for SBP in time $\quad 15$

5.1 Pseudo-spectral operators . . . . . . . . . . . . . . . 16

5.2 Centered finite-difference operators . . . . . . . . . . . . . . 17

5.2.1 Proof for second-order accurate operators . . . . . . . 18

6 Dual time-stepping $\quad 21$

6.1 The classical dual time-stepping . . . . . . . . . . . . . . . 21

6.2 The second-derivative DTS technique . . . . . . . . . . . . 22

6.3 A numerical comparison . . . . . . . . . . . . . . . . 23

6.4 The main drawback and aftermath . . . . . . . . . . . 24 
7 Multi-grid techniques $\quad 25$

7.1 Multi-grid for parabolic problems . . . . . . . . . . . . . 26

7.1.1 The Galerkin condition . . . . . . . . . . . . . 27

7.1.2 SBP-preserving interpolation operators . . . . . . . . 27

7.2 Multi-grid for hyperbolic problems . . . . . . . . . . . . . . 29

7.2.1 The TVD-MG algorithm . . . . . . . . . . . . . 30

7.2.2 Extension to higher order methods and systems . . . . . . 31

8 Summary of papers 33

References $\quad 35$

I. On conservation and stability properties for summation-by-parts schemes . . . . . . . . . . . . . . . . . 39

II. On pseudo-spectral time discretizations in summation-by-parts form 55

III. Eigenvalue analysis for summation-by-parts finite difference time discretizations ...................... 67

IV. Dual time-stepping using second derivatives . . . . . . . . . . 105

V. A new multigrid formulation for high order finite difference methods on summation-by-parts form . . . . . . . . . . . . . . . . 129

VI. Multigrid schemes for high order discretizations of hyperbolic problems ......................... 155 


\section{Introduction}

The process of computing the solution to scientific problems can be divided into three phases: the modeling, the approximation and the solution phase. To start with, a problem (often) arising from a physical or chemical phenomenon is studied and formulated mathematically. Assuming that this mathematical model adheres to reality, its solution can be used to predict the behavior of the phenomenon in question. Moreover, if the solution to this problem exists, is unique and its behavior changes continuously with the given data, the mathematical model is said to be well-posed [16].

Since most mathematical problems can not be solved exactly, the modeling phase is usually followed by the approximation step. The first part of this thesis is devoted to this branch of computational mathematics. In particular, high order numerical approximations of well-posed differential problems mimicking the integration-by-parts property are studied $[21,10]$. These discrete problems are said to be in summation-by-parts (SBP) form and can be formulated in different ways. Various SBP approximations in space and time are studied as well as the properties that these retain from the mathematical model. Particular attention is given throughout this thesis to energy-stability [14].

Once an accurate approximation of the mathematical formulation is obtained, efficient algorithms to find the solution are required. The second and last part of this thesis deals with that part and consists of convergence acceleration techniques for high order summation-by-parts approximations. In particular, the Dual Time-Stepping (DTS) and the Multi-Grid (MG) techniques are used to compute steady-state solutions for systems of equations obtained from numerical approximations of parabolic and hyperbolic problems.

The thesis is organised as follows: in Chapter 2, the concepts of conservation, energy boundedness and well-posedness of numerical approximations are presented. In Chapter 3, the different kinds of SBP operators used in the articles are introduced. Chapter 4 deals with a comparison of different SBP approximations for the variable coefficient linear advection equation. In Chapter 5, the eigenvalue analysis of SBP time approximations based on pseudo-spectral and finite-difference methods is discussed. The DTS and MG procedures are presented and analyzed in Chapter 6 and 7 , respectively. 



\section{Conservation, energy boundedness and well-posedness}

To illustrate the concepts of conservation, energy boundedness and well-posedness [14], consider the following initial-boundary value problem (IBVP) on conservation form

$$
\begin{aligned}
& u_{t}+f_{x}=0, \quad \alpha<x<\beta, \quad t>0, \\
& u=h(x), \quad \alpha<x<\beta, \quad t=0, \\
& B_{\alpha} u=g_{\alpha}(t), \quad x=\alpha, \quad t>0, \\
& B_{\beta} u=g_{\beta}(t), \quad x=\beta, \quad t>0 .
\end{aligned}
$$

Here, $f=f(u, x)$ is the flux function and $B_{\alpha}, B_{\beta}$ are the left and right boundary operators, respectively. Moreover, the initial data $h$ and boundary data $g_{\alpha}, g_{\beta}$ are given functions.

We introduce the scalar product and induced norm in $L^{2}(\alpha, \beta)$

$$
(v, w)_{2}=\int_{\alpha}^{\beta} v w \mathrm{~d} x, \quad\|v\|_{2}=\sqrt{(v, v)_{2}} .
$$

The problem (2.1) is said to be in conservative form since the integral of the solution $u$ changes only by the flux through the boundaries, namely

$$
\frac{\mathrm{d}}{\mathrm{d} t}(1, u)_{2}=f(u(\alpha, t), \alpha)-f(u(\beta, t), \beta) .
$$

Furthermore, the problem (2.1) is energy-bounded if its solution depends continuously on the data, i.e. if the estimate

$$
\|u(\cdot, t)\|_{2}^{2} \leq K(t)\left[\|h\|_{2}^{2}+\int_{0}^{t} g_{\alpha}(\tau)+g_{\beta}(\tau) \mathrm{d} \tau\right]
$$

holds with $K(t)$ independent of the data and bounded for finite times. If, in addition to the energy-boundedness, a unique solution for the IBVP (2.1) exists, this problem is said to be well-posed [16]. Existence is guaranteed by using a minimal number of boundary conditions, while uniqueness directly follows from (2.4) if the problem is linear.

Remark 2.0.1. Energy boundedness can be extended to non-linear and nonconservative problems with a forcing function. 
As an example, in Paper I we have shown that the advection problem with a variable coefficient

$$
\begin{aligned}
& u_{t}+(a u)_{x}=0, \quad \alpha<x<\beta, \quad t>0, \\
& u=h(x), \quad \alpha<x<\beta, \quad t=0, \\
& u=g_{\alpha}(t), \quad x=\alpha, \quad t>0,
\end{aligned}
$$

is both conservative and energy-bounded, if $a(\alpha)>0$ and $a(\beta)>0$. In particular, by integrating $(2.5)$ over $[\alpha, \beta]$ we found the conservation relation

$$
\frac{\mathrm{d}}{\mathrm{d} t}(1, u)_{2}=a(\alpha) g_{\alpha}(t)-a(\beta) u(\beta, t) .
$$

By using the energy-method (multiplying by $u$ and integrating over $[\alpha, \beta]$ ), we also found

$$
\frac{\mathrm{d}}{\mathrm{d} t}\|u\|_{2}^{2}=a(\alpha) g_{\alpha}^{2}(t)-a(\beta) u^{2}(\beta, t)-\left(u, a_{x} u\right)_{2} .
$$

If $a_{x} \in L^{\infty}(\alpha, \beta)$, this relation leads to the estimate

$$
\|u(\cdot, t)\|_{2}^{2} \leq e^{\left\|a_{x}\right\|_{\infty} t}\left[\|h\|_{2}^{2}+\int_{0}^{t} e^{-\left\|a_{x}\right\|_{\infty} \tau}\left(a(\alpha) g_{\alpha}^{2}(\tau)-a(\beta) u^{2}(\beta, \tau)\right) \mathrm{d} \tau\right],
$$

with $\left\|a_{x}\right\|_{\infty}=\max _{x \in[\alpha, \beta]}\left|a_{x}(x)\right|$, which proves the energy boundedness of (2.1). 


\section{Summation-By-Parts operators}

Numerical approximations are needed due to the lack of closed-form solutions to most IBVPs. These approximations, henceforth referred to as discrete problems, can be formulated in different ways and each of these leads to a specific loss of information. As an example, consider the translation of a sentence from one language to another. Since it is impossible to establish a one-to-one correspondence between two different languages, any translation is unavoidably affected by the criterion used, i.e. either preserving the meaning of the original sentence or translating it word-for-word. Likewise, numerical approximations can preserve some, but not all the properties of the original problem.

The conservation relation can be obtained from the IBVP by integrating over the spatial domain, whereas the energy estimate also requires the product rule and integration-by-parts property to hold. SBP operators are approximations to the first and second derivative which both include a quadrature formula that approximates integration and mimic the integration-by-parts rule. Since we are interested in preserving both conservation and energy-boundedness, we henceforth consider discrete formulations involving SBP operators.

\subsection{Finite-difference approximations}

SBP operators were originally introduced for centered finite difference methods on equidistant grids [21]. Consider a uniform mesh of $N+1$ grid points on the interval $[\alpha, \beta]$ with $x_{j}=\alpha+j \Delta x$, where $j=0, \ldots, N$ and $\Delta x=(\beta-\alpha) / N$ is the spatial step. Henceforth, we denote discrete approximations of a real-valued function $v(x), x \in[\alpha, \beta]$, with the vector $\mathbf{v}=\left[v_{0}, \ldots, v_{N}\right]^{T}$, where $v_{j} \approx v\left(x_{j}\right)$.

\subsubsection{Centered finite-differences}

A centered finite-difference $(p, q)$-accurate approximation of the first derivative with the SBP property is a discrete operator $D=P^{-1} Q$ such that [39]

i) its truncation error is $\mathcal{O}\left(\Delta x^{p}\right)$ in the interior and $\mathcal{O}\left(\Delta x^{q}\right)$ at the boundaries,

ii) $P$ is a symmetric positive definite matrix, 
iii) $Q+Q^{T}=B=\operatorname{diag}(-1,0, \ldots, 0,1)$.

Condition ii) defines a scalar product and a norm which are discrete counterparts to $(2.2)$ in $L^{2}(\alpha, \beta)$, i.e.

$$
(\mathbf{v}, \mathbf{w})_{P}=\mathbf{v}^{T} P \mathbf{w}, \quad \quad\|\mathbf{v}\|_{P}=\sqrt{(\mathbf{v}, \mathbf{v})_{P}} .
$$

Moreover, condition iii) causes $D$ to exactly mimic the integration-by-parts rule

$$
\left(v, w_{x}\right)_{2}=v(\beta) w(\beta)-v(\alpha) w(\alpha)-\left(v_{x}, w\right)_{2}
$$

as

$$
(\mathbf{v}, D \mathbf{w})_{P}=v_{N} w_{N}-v_{0} w_{0}-(D \mathbf{v}, \mathbf{w})_{P} .
$$

Centered finite-difference based SBP operators are available for even orders $p=2 q$ in the interior. We will for stability reasons [40, 12, 32] only consider operators with a diagonal norm $P$. To ease the notation, unless otherwise specified we refer to the order of these approximations by the order of the SBP operators on the interior nodes. As an example, the second order first derivative SBP operator is given by

$$
P=\Delta x \operatorname{diag}\left(\frac{1}{2}, 1, \ldots, 1, \frac{1}{2}\right), \quad Q=\left[\begin{array}{ccccc}
-\frac{1}{2} & \frac{1}{2} & & & \\
-\frac{1}{2} & 0 & \frac{1}{2} & & \\
& \ddots & \ddots & \ddots & \\
& & -\frac{1}{2} & 0 & \frac{1}{2} \\
& & & -\frac{1}{2} & \frac{1}{2}
\end{array}\right] .
$$

Second derivative SBP operators can be defined by applying the first derivative operator twice as $D_{2}=D^{2}$. However, these operators do not modify modes with the highest frequency that can exist on the grid and hence can not be used for multi-grid methods (see Remark 7.1.4). This issue is solved by using compact second derivative SBP operators.

A $2 q$ th order accurate approximation for the compact second derivative based on a diagonal norm can be written as $D_{2}=P^{-1}\left(-S^{T} A+B\right) S$ such that $[9,27]$

i) its truncation error is $\mathcal{O}\left(\Delta x^{2 q}\right)$ in the interior and $\mathcal{O}\left(\Delta x^{q}\right)$ at the boundaries,

ii) $S$ is an approximation of the first derivative at the boundaries with a truncation error $\mathcal{O}\left(\Delta x^{q+1}\right)$,

iii) $A+A^{T}$ is positive semidefinite.

The SBP operator $D_{2}$ mimics the integration-by-parts rule for the second derivative

$$
\left(v, w_{x x}\right)_{2}=v(\beta) w_{x}(\beta)-v(\alpha) w_{x}(\alpha)-\left(v_{x}, w_{x}\right)_{2}
$$


as

$$
\left(\mathbf{v}, D_{2} \mathbf{w}\right)_{P}=v_{N}(S \mathbf{w})_{N}-v_{0}(S \mathbf{w})_{0}-(S \mathbf{v}, S \mathbf{w})_{A} .
$$

Here the notation $(\cdot, \cdot)_{A}$ does not represent a discrete scalar product due to the semidefiniteness of $A+A^{T}$ and $A$ not being symmetric. Furthermore, $(S \mathbf{v}, S \mathbf{w})_{A}$ does not necessarily approximate $\left(v_{x}, w_{x}\right)_{2}$. However, condition iii) is sufficient to preserve the energy-boundedness of IBVPs involving second derivatives.

Remark 3.1.1. The second-derivative $S B P$ operators $D_{2}=D^{2}$ can be obtained from the previous definition by considering $S=D_{1}$ and $A=D_{1}^{T} P D_{1}$. In this case, the integration-by-parts rule for the second derivative (3.3) is perfectly mimicked and the matrix $A$ is symmetric. The additional constraint $A=A^{T}$ is required, for example, to approximate the wave equation.

The second order compact SBP operator approximating the second derivative is

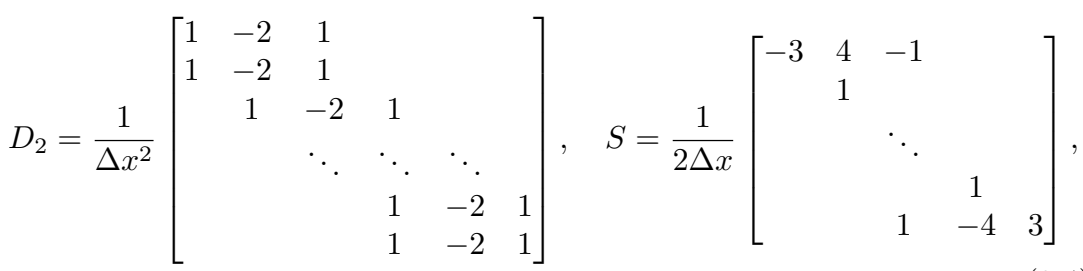

with the same norm $P$ as in (3.2).

Remark 3.1.2. In Paper $V$, we also use compact $S B P$ approximations for the second derivative with a variable coefficient, i.e. $\left(\varepsilon(x) u_{x}\right)_{x}$. For details on these discrete operators, see [24].

\subsubsection{Upwind difference operators}

Flow problems approximated with central finite difference stencils may introduce spurious oscillations. To address this problem, the addition of stable and accurate artificial dissipation operators [29] is often necessary. However, artificial dissipation compatible with high order accurate SBP operators can be difficult to apply in practice. In Paper VI, we rather use discrete operators based on non-centered finite difference stencils which naturally introduce upwinding and satisfy a modified SBP property.

The difference operators $D_{+}=P^{-1}\left(Q_{+}+\frac{B}{2}\right)$ and $D_{-}=P^{-1}\left(Q_{-}+\frac{B}{2}\right)$ are $p$ th order diagonal-norm upwind SBP operators for the first derivative if

i) the truncation error is $\mathcal{O}\left(\Delta x^{p}\right)$ in the interior and $\mathcal{O}\left(\Delta x^{\lfloor p / 2\rfloor}\right)$ at the boundaries, 
ii) $P$ is a symmetric positive definite matrix,

iii) $Q_{+}+Q_{-}^{T}=0$,

iv) $Q_{+}+Q_{+}^{T}\left(Q_{-}+Q_{-}^{T}\right)$ is positive (negative) semidefinite.

These approximations of the first derivative exactly mimic the integration-byparts rule (3.1) as

$$
\left(\mathbf{v}, D_{+} \mathbf{w}\right)_{P}=v_{N} w_{N}-v_{0} w_{0}-\left(D_{-} \mathbf{v}, \mathbf{w}\right)_{P} .
$$

The upwind SBP operators were originally derived from second up to ninth order [25]. In Paper VI, we also introduce first order SBP upwind discretizations given (here for the positive variant) by

$$
P=\Delta x \operatorname{diag}(1, \ldots, 1), \quad Q_{+}=\left[\begin{array}{cccc}
\frac{1}{2} & & & \\
-1 & 1 & & \\
& \ddots & \ddots & \\
& & -1 & \frac{1}{2}
\end{array}\right] .
$$

\subsection{Pseudo-spectral collocation methods}

SBP operators were extended to grids non-conforming to the boundaries in [10], where it was shown that first derivative SBP approximations can be obtained by starting from a quadrature rule. In Paper I, these operators are referred to as Generalized Summation-By-Parts (GSBP) operators.

Consider a set of nodes $\mathbf{x}=\left[x_{0}, \ldots, x_{N}\right]^{T}$, with $x_{0}<\ldots<x_{N}$, that may not include one or both boundary nodes. An operator $D=P^{-1} Q$ is a $p$ th order accurate approximation of the first derivative with the GSBP property if

i) $D \mathbf{x}^{j}=P^{-1} Q \mathbf{x}^{j}=j \mathbf{x}^{j-1}, j \in\{0, \ldots, p\}$,

ii) $P$ is a symmetric positive definite matrix,

iii) $Q+Q^{T}=E$, where $\left(\mathbf{x}^{i}\right)^{T} E \mathbf{x}^{j}=\beta^{i+j}-\alpha^{i+j}, i, j \in\{0, \ldots, r\}, r \geq p$.

To better understand the connection between SBP and GSBP operators, we first compare condition i) of the two definitions. For $p$ th-order accurate SBP operators based on finite-difference methods, the monomials $x^{j}$ are exactly differentiated for $j \in\{0, \ldots, p\}$ at the interior nodes, as in the definition of GSBP operators. However, for GSBP operators the exactness holds also at the boundary nodes.

Another important distinction between the two definitions lies in condition iii). The matrix $E$ can be written in terms of boundary interpolants of degree $r$, i.e. $\mathbf{t}_{\alpha}$ and $\mathbf{t}_{\beta}$ such that

$$
\mathbf{t}_{\phi}^{T} \mathbf{u} \approx u(\phi): \quad \mathbf{t}_{\phi}^{T} \mathbf{x}^{j}=\phi^{j}, \quad j \in\{0, \ldots, r\}, \quad \phi \in\{\alpha, \beta\} .
$$


This gives rise to $E=\mathbf{t}_{\beta} \mathbf{t}_{\beta}^{T}-\mathbf{t}_{\alpha} \mathbf{t}_{\alpha}^{T}$ and to the following mimicking of the integration-by-parts rule (3.1)

$$
(\mathbf{v}, D \mathbf{w})_{P}=\left(\mathbf{t}_{\beta}^{T} \mathbf{v}\right)\left(\mathbf{t}_{\beta}^{T} \mathbf{w}\right)-\left(\mathbf{t}_{\alpha}^{T} \mathbf{v}\right)\left(\mathbf{t}_{\alpha}^{T} \mathbf{w}\right)-(D \mathbf{v}, \mathbf{w})_{P} .
$$

Also for SBP operators based on finite-difference methods, the matrix $B$ can be expressed in terms of boundary interpolants, i.e. $B=\mathbf{e}_{\beta} \mathbf{e}_{\beta}^{T}-\mathbf{e}_{\alpha} \mathbf{e}_{\alpha}^{T}$ with $\mathbf{e}_{\alpha}=[1,0, \ldots, 0]^{T}$ and $\mathbf{e}_{\beta}=[0, \ldots, 0,1]^{T}$. In this case, both the interpolants are exact and $r=\infty$.

In Paper II, we analyzed diagonal norm GSBP operators based on pseudospectral collocation methods. In particular, we considered approximations for the first derivative based on the Legendre-Gauss-Lobatto (henceforth denoted by LGL, with both boundary nodes and $p=2 N-1$ ), Legendre-Gauss-Radau (denoted by LGR, with one boundary node and $p=2 N$ ) and Legendre-Gauss (LG, no boundary node and $p=2 N+1$ ) quadratures.

Remark 3.2.1. Since LGL quadrature includes both boundary nodes, the GSBP operators based on the LGL quadrature are actually an example of SBP operators which are not based on centered finite differences. To avoid ambiguity, henceforth in this thesis only the discretizations not including one or both boundary nodes are referred to as GSBP approximations.

For pseudo-spectral methods, the grid is usually the reference interval $[\alpha, \beta]=$ $[-1,1]$. As an example, the second order accurate GSBP operators based on the LGR quadrature are defined on the three-point grid $\mathbf{x}=[-1, \quad(1-\sqrt{6}) / 5$, $(1+\sqrt{6}) / 5]^{T}$. In this case, the first derivative is approximated by $D=P^{-1} Q$ with

$$
\begin{gathered}
P=\frac{1}{18} \operatorname{diag}(4,16+\sqrt{6}, 16-\sqrt{6}), \\
Q=\frac{1}{108}\left[\begin{array}{ccc}
-48 & 24+14 \sqrt{6} & 24-14 \sqrt{6} \\
-12-32 \sqrt{6} & 87-18 \sqrt{6} & -75+50 \sqrt{6} \\
-12+32 \sqrt{6} & -75-50 \sqrt{6} & 87-18 \sqrt{6}
\end{array}\right] .
\end{gathered}
$$

For these operators, the boundary interpolants fulfilling condition iii) are

$$
\mathbf{t}_{\alpha}=\mathbf{e}_{\alpha}=[1,0,0]^{T}, \quad \mathbf{t}_{\beta}=\left[\frac{1}{3}, \frac{2-3 \sqrt{6}}{6}, \frac{2+3 \sqrt{6}}{6}\right]^{T} .
$$

\subsection{Multi-dimensional SBP operators}

Approximations of partial derivatives in multi-dimensional settings can in a straightforward manner be constructed as Kronecker products of one-dimensional SBP operators. The Kronecker product of two matrices $A=\left\{a_{i j}\right\} \in \mathbb{R}^{m \times n}$ and $B \in \mathbb{R}^{r \times s}$ is given by

$$
A \otimes B=\left[\begin{array}{ccc}
a_{11} B & \ldots & a_{1 n} B \\
\vdots & \ddots & \vdots \\
a_{m 1} B & \ldots & a_{m n} B
\end{array}\right] \in \mathbb{R}^{m r \times n s} .
$$


Consider a two-dimensional grid of $N \times M$ nodes. The SBP operators for the partial derivatives on $x$ and $y$ can be written as

$$
\frac{\partial}{\partial x} \approx\left(P_{x}^{-1} Q_{x} \otimes I_{M}\right), \quad \frac{\partial}{\partial y} \approx\left(I_{N} \otimes P_{y}^{-1} Q_{y}\right),
$$

where the subscripts $x, y$ refer to the coordinate directions and $I_{K} \in \mathbb{R}^{K \times K}$ denotes the identity matrix.

Remark 3.3.1. The Kronecker product can also be used to write approximations for systems of equations, as was done in Paper II, IV and VI. 


\section{Conservation and stability for SBP-SAT approximations}

In this chapter, we will discuss spatial approximations of the variable coefficient advection equation (2.5) with SBP and GSBP operators. To preserve both conservation and energy-boundedness of the original problem, (G)SBP operators are complemented with Simultaneous-Approximation-Terms (SAT) [41] weakly imposing the boundary conditions, although other options exist [34, 13, 28, 35]. For semi-discrete formulations, the energy-boundedness is henceforth referred to as energy-stability [14].

\subsection{The splitting technique}

We start by considering numerical approximations based on diagonal norm SBP operators. A straightforward semi-discrete formulation of (2.5) with the SBPSAT approach gives

$$
\mathbf{u}_{t}+P^{-1} Q A \mathbf{u}=\sigma_{\alpha} A P^{-1} \mathbf{e}_{\alpha}\left(\mathbf{e}_{\alpha}^{T} \mathbf{u}-g_{\alpha}(t)\right),
$$

where $A=\operatorname{diag}\left(a\left(x_{0}\right), \ldots, a\left(x_{N}\right)\right)$ and $\sigma_{\alpha} \in \mathbb{R}$ is a penalty parameter which can be tuned for conservation and stability. By multiplying (4.1) from the left by $\mathbf{1}^{T} P$, with $\mathbf{1}=[1, \ldots, 1]^{T}$ and considering $\sigma_{\alpha}=-1$, we find

$$
\frac{\mathrm{d}}{\mathrm{d} t}(\mathbf{1}, \mathbf{u})_{P}=a(\alpha) g_{\alpha}(t)-a(\beta) u_{N}
$$

This relation mimics (2.6) perfectly and hence (4.1) is conservative. However, in [32] it was shown that this formulation can not be energy-stable. This is due to the fact that the product rule, needed to prove the energy-boundedness of (2.5), is not mimicked by first derivative SBP operators.

In Paper I, we prove that

$$
\mathbf{u}_{t}+\frac{1}{2}(D A+A D) \mathbf{u}+\frac{1}{2} U D \mathbf{a}=\sigma_{\alpha} A P^{-1} \mathbf{e}_{\alpha}\left(\mathbf{e}_{\alpha}^{T} \mathbf{u}-g_{\alpha}(t)\right)
$$

is a conservative and stable spatial approximation of $(2.5)$ for $\sigma_{\alpha}=-1$. In this formulation, we used $U=\operatorname{diag}\left(u_{0}, \ldots, u_{N}\right)$ and $\mathbf{a}=A \mathbf{1}$. But most importantly, in (4.2) we introduced a splitting of $(a u)_{x}=\frac{1}{2} a_{x} u+\frac{1}{2}\left[(a u)_{x}+a u_{x}\right]$ into 
symmetric and anti-symmetric contributions

$$
\frac{1}{2} a_{x} u \approx \frac{1}{2} U D \mathbf{a}, \quad \frac{1}{2}\left[(a u)_{x}+a u_{x}\right] \approx \frac{1}{2}(D A+A D) \mathbf{u} .
$$

This splitting guarantees that both the symmetric and anti-symmetric portions, after multiplication by a smooth function $v$ and summation over the spatial domain, act as in the continuous setting. It is straightforward to check that the symmetric part fulfills

$$
\frac{1}{2}\left(u a_{x}, v\right)_{2}=\frac{1}{2}\left(v a_{x}, u\right)_{2}, \quad \frac{1}{2}(U D \mathbf{a}, \mathbf{v})_{P}=\frac{1}{2}(V D \mathbf{a}, \mathbf{u})_{P},
$$

with $V=\operatorname{diag}\left(v_{0}, \ldots, v_{N}\right)$. On the other hand, the anti-symmetric part gives rise to the integration-by-parts rule

$$
\frac{1}{2}\left((a u)_{x}+a u_{x}, v\right)_{2}=\left.a u v\right|_{\beta}-\left.a u v\right|_{\alpha}-\frac{1}{2}\left((a v)_{x}+a v_{x}, u\right)_{2}
$$

which is perfectly mimicked by

$$
\frac{1}{2}(D(A \mathbf{u})+A D \mathbf{u}, \mathbf{v})_{P}=a_{N} u_{N} v_{N}-a_{0} u_{0} v_{0}-\frac{1}{2}(D(A \mathbf{v})+A D \mathbf{v}, \mathbf{u})_{P} .
$$

Recalling that conservation and energy-boundedness can be proved by multiplying (2.5) from the left by $\mathbf{v}^{T} P$ with $\mathbf{v}=\mathbf{1}$ and $\mathbf{v}=\mathbf{u}$ respectively, we conclude that mimicking (4.3) is the key to provide both conservation and energystability.

\subsection{GSBP-SAT approximations}

In Paper I, we show that conservative and energy-stable approximations for variable coefficient problems require a boundary conforming grid and exact numerical mimicking of integration-by-parts. In other words, GSBP-SAT approximations can not be both conservative and energy-stable because (4.4) does not hold. If $D$ is a GSBP operator, one can instead prove

$$
\begin{aligned}
\frac{1}{2}(D(A \mathbf{u})+A D \mathbf{u}, \mathbf{v})_{P} & =\frac{1}{2}\left[\left(\mathbf{t}_{\beta}^{T} A \mathbf{u}\right)\left(\mathbf{t}_{\beta}^{T} \mathbf{v}\right)+\left(\mathbf{t}_{\beta}^{T} A \mathbf{v}\right)\left(\mathbf{t}_{\beta}^{T} \mathbf{u}\right)\right] \\
& -\frac{1}{2}\left[\left(\mathbf{t}_{\alpha}^{T} A \mathbf{u}\right)\left(\mathbf{t}_{\alpha}^{T} \mathbf{v}\right)+\left(\mathbf{t}_{\alpha}^{T} A \mathbf{v}\right)\left(\mathbf{t}_{\alpha}^{T} \mathbf{u}\right)\right] \\
& -\frac{1}{2}(D(A \mathbf{v})+A D \mathbf{v}, \mathbf{u})_{P} .
\end{aligned}
$$

Here, the boundary terms consistently approximate both $a(\alpha) u(\alpha) v(\alpha)$ and $a(\beta) u(\beta) v(\beta)$. However, the interpolation operators $\mathbf{t}_{\alpha}$ and $\mathbf{t}_{\beta}$ may not preserve the sign of the boundary terms, leading to instabilities. The relation (4.5) suggests a possible strategy to restore conservation and energy-stability for GSBP-SAT approximations. 
In Paper I, we introduced modified GSBP operators fulfilling (4.4). Rather than approximating the first derivative, these operators discretize the anti-symmetric portion of $(a u)_{x}$ as

$$
P^{-1} \Theta \mathbf{u} \approx \frac{1}{2}\left[(a u)_{x}+a u_{x}\right] .
$$

In particular, $P^{-1} \Theta$ is a $p$ th order accurate approximation of the anti-symmetric portion of $(a u)_{x}$ if

i) $P^{-1} \Theta \mathbf{x}^{j}=(1 / 2) A_{x} \mathbf{x}^{j}+j A \mathbf{x}^{j-1}, j \in\{0, \ldots, p\}$,

ii) $P$ is a symmetric positive definite matrix,

iii) $\Theta+\Theta^{T}=a(\beta) \mathbf{t}_{\beta} \mathbf{t}_{\beta}^{T}-a(\alpha) \mathbf{t}_{\alpha} \mathbf{t}_{\alpha}^{T}$,

where $A_{x}=\operatorname{diag}\left(a_{x}\left(x_{0}\right), \ldots, a_{x}\left(x_{N}\right)\right)$. These approximations for the antisymmetric part of $(a u)_{x}$, named $a$-Generalized Summation-By-Parts ( $a$-GSBP) operators, consistently represent also its symmetric part as

$$
U P^{-1} \Theta \mathbf{1} \approx \frac{1}{2} a_{x} u .
$$

It was shown that $a$-GSBP operators naturally lead to both conservation and energy-stability of spatial approximations of (2.5).

Remark 4.2.1. Another approach to make pseudo-spectral GSBP-SAT approximations conservative and stable is to use a modified coefficient instead of a $(x)$. If a grid with $N+1$ points is used, the function a $(x)$ is evaluated at another set of $N+1$ points including the boundary nodes, such as LGL nodes. Afterwards, the unique $N$ th degree interpolating polynomial is used to produce the diagonal matrix $A$ in the GSBP-SAT discretization. This procedure allows for a correct representation of the coefficient at the boundaries and leads to both conservation and energy-stability, if additional care is taken to construct SATs. For further details on this approach, see [36]. 



\section{The eigenvalues for SBP in time}

The SBP-SAT technique can also be used to approximate initial value problems

$$
\begin{aligned}
u_{t}+\lambda u & =0, \quad 0<t<T \\
u(0) & =f .
\end{aligned}
$$

Time integration methods based on finite-difference SBP operators were first introduced in [33]. Here, it was shown that diagonal-norm dual-consistent [4, 18] $2 p$ th-order accurate approximations of the first derivative lead to $2 p$ th-order accurate solutions. Moreover, in [23] SBP in time was proved to be $A$-, $L$ - and $B$ stable [17]. In [5], these results were proved also for GSBP-SAT approximations of (5.1). It was also shown that dual-consistent (G)SBP time-marching methods can be interpreted as Runge-Kutta methods. Additional stability properties of time integration methods based on (G)SBP operators were studied in [37, 22].

Most of these theoretical findings are based on the following

Assumption 5.0.1. The operator $\mathcal{D}=P^{-1}\left(Q-\sigma \mathbf{t}_{\alpha} \mathbf{t}_{\alpha}^{T}\right)$ has eigenvalues with strictly positive real parts for $\sigma<-1 / 2$.

Remark 5.0.1. SBP-SAT approximations in time fulfilling Assumption 5.0.1 lead to invertible systems when energy-stable spatial approximations are used.

In this thesis, we discuss Assumption 5.0.1 for both pseudo-spectral GSBP (Paper II) and centered finite-difference SBP operators (Paper III). Our arguments make extensive use of the following theorem $[8,7]$

Theorem 5.0.1. Given a matrix $A$, suppose that $H=\frac{1}{2}\left(G A+A^{T} G\right)$ and $S=\frac{1}{2}\left(G A-A^{T} G\right)$ for some positive definite matrix $G$ and some positive semidefinite matrix $H$. Then $A$ has eigenvalues with strictly positive real parts if, and only if, no eigenvector of $G^{-1} S$ lies in the nullspace of $H$.

Proving Assumption 5.0.1 by means of Theorem 5.0.1 is not straightforward, since $\mathcal{D}$ depends on the parameter $\sigma$, the dimension of the matrix $N$, the order of accuracy and the grid nodes $\mathbf{x}$ (or the mesh-size $\Delta x$ ). In this section, we sketch the proof for both pseudo-spectral and centered finite-difference operators. 


\subsection{Pseudo-spectral operators}

In Paper II, we prove Assumption 5.0.1 for the pseudo-spectral GSBP operators $\mathcal{D} \in \mathbb{R}^{(N+1) \times(N+1)}$ based on LGL, LGR and LG quadrature rules. Firstly, we recall that $D=P^{-1} Q$ is similar to [38]

$$
\widehat{D}=\left[\begin{array}{cccccc}
0 & 1 & 0 & 1 & 0 & \cdots \\
0 & 0 & 3 & 0 & 3 & \ddots \\
0 & 0 & 0 & 5 & 0 & \ddots \\
0 & 0 & 0 & 0 & 7 & \ddots \\
0 & 0 & 0 & 0 & 0 & \ddots \\
\vdots & \ddots & \ddots & \ddots & \ddots & \ddots
\end{array}\right] \in \mathbb{R}^{(N+1) \times(N+1)} .
$$

This result can be proved by considering an arbitrary set of $N+1$ grid nodes $\mathbf{x}$ in the interval $[-1,1]$, and the Legendre basis functions $\phi_{n}(x)$ with $n=0, \ldots, N$, given by

$$
\phi_{0}(x)=1, \quad \phi_{1}(x)=x, \quad \phi_{2}(x)=\frac{1}{2}\left(3 x^{2}-1\right), \quad \phi_{3}(x)=\frac{1}{2}\left(5 x^{3}-3 x\right), \ldots
$$

Let $\phi_{i}=\phi_{i}(\mathbf{x})$ be the grid function corresponding to the $i$ th degree Legendre polynomial and consider the Vandermonde matrix $V=\left[\phi_{0}, \phi_{1}, \phi_{2}, \phi_{3}, \ldots\right]$. Since the first derivative is exactly mimicked on the grid monomials $\mathbf{x}^{j}, j=$ $0, \ldots, N$, we can write

$$
D V=\left[\mathbf{0}, \mathbf{1}, 3 \mathbf{x}, \frac{3}{2}\left(5 \mathbf{x}^{2}-\mathbf{1}\right), \ldots\right]=\left[\mathbf{0}, \boldsymbol{\phi}_{0}, 3 \boldsymbol{\phi}_{1}, \boldsymbol{\phi}_{0}+5 \boldsymbol{\phi}_{2}, \ldots\right]=V \widehat{D}
$$

and $D=V \widehat{D} V^{-1}$. Hence, $D$ and $\widehat{D}$ are similar and have the same eigenvalues. Note that $\widehat{D}$ does not depend on the grid nodes $\mathbf{x}$.

Secondly, we noted the following

Remark 5.1.1. Assumption 5.0.1 holds for $\mathcal{D}$ if, and only if, it holds for $\mathcal{D}^{-1}$.

Thirdly, we proved that for GSBP operators based on pseudo-spectral collocation methods $\mathcal{D}=P^{-1}\left(Q-\sigma \mathbf{t}_{\alpha} \mathbf{t}_{\alpha}^{T}\right)$ is invertible for $\sigma \neq 0$. The matrix $\mathcal{D}^{-1}$ is similar to

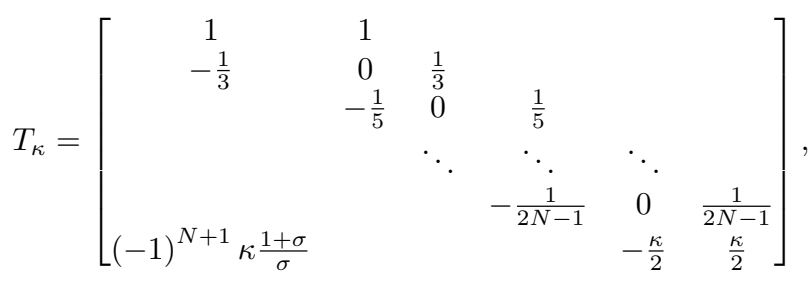


with

$$
\kappa= \begin{cases}2 /(2 N+1), & \text { for LGR and LG, } \\ 2 / N, & \text { for LGL. }\end{cases}
$$

As a result, if $T_{k}$ has eigenvalues with strictly positive real parts, then Assumption 5.0.1 is fulfilled due to Remark 5.1.1.

Fourthly, we apply Theorem 5.0.1 to $A=T_{\kappa}$ and $G=\operatorname{diag}(1,3, \ldots, 2 N-1$, $2 / \kappa)$. The auxiliary matrix

$$
H=\frac{1}{2}\left(G T_{\kappa}+T_{\kappa}^{T} G\right)=\left[\begin{array}{cccccc}
1 & 0 & \ldots & 0 & (-1)^{N+1} \frac{1+\sigma}{\sigma} \\
0 & 0 & \ldots & 0 & 0 \\
\vdots & \vdots & \ldots & \vdots & \vdots \\
0 & 0 & \ldots & 0 & 0 \\
(-1)^{N+1} \frac{1+\sigma}{\sigma} & 0 & \ldots & 0 & 1
\end{array}\right]
$$

is positive semidefinite for $\sigma<-1 / 2$ and its nullspace is given by all the vectors $\mathbf{v}=\left[v_{0}, v_{1}, \ldots, v_{N-1}, v_{N}\right]^{T}$ where $v_{0}=v_{N}=0$.

Finally, since the vectors belonging to the nullspace of $H$ can not be eigenvectors to

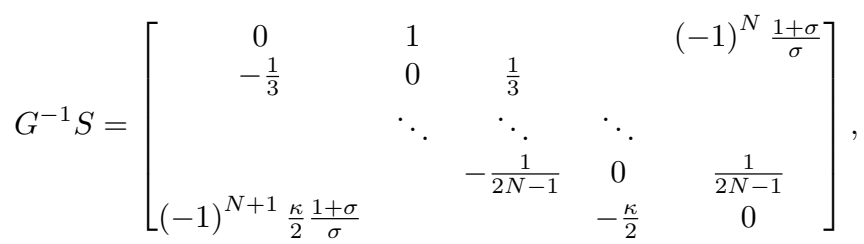

the matrix $T_{\kappa}$ has eigenvalues with strictly positive real parts. Therefore, Assumption 5.0.1 is fulfilled by GSBP operators based on LGL, LGR and LG pseudo-spectral collocation methods.

\subsection{Centered finite-difference operators}

The proof for finite-difference based SBP-SAT approximations is much more technical since these operators can not be rewritten in terms of a modal basis.

To start with, we directly apply Theorem 5.0.1 to $\mathcal{D}=P^{-1}\left(Q-\sigma \mathbf{e}_{0} \mathbf{e}_{0}^{T}\right)$ with $G=P$. The matrix

$$
H=\frac{1}{2}\left(P \mathcal{D}+\mathcal{D}^{T} P\right)=\frac{1}{2}\left[\mathbf{e}_{N} \mathbf{e}_{N}^{T}-(1+2 \sigma) \mathbf{e}_{0} \mathbf{e}_{0}^{T}\right]
$$

is positive semidefinite for $\sigma<-1 / 2$ and its nullspace is given by all the vectors $\mathbf{v}=\left[v_{0}, v_{1}, \ldots, v_{N-1}, v_{N}\right]^{T}$ where $v_{0}=v_{N}=0$. If such vectors do not belong to the nullspace of $P^{-1} S$, with

$$
S=\frac{1}{2}\left(P \mathcal{D}-\mathcal{D}^{T} P\right)=Q-\frac{1}{2} \mathbf{e}_{N} \mathbf{e}_{N}^{T}+\frac{1}{2} \mathbf{e}_{0} \mathbf{e}_{0}^{T},
$$

then $\mathcal{D}$ has eigenvalues with strictly positive real parts. Since it can be proved that $P^{-1} S$ has only imaginary eigenvalues, the following lemma holds [33]. 
Lemma 5.2.1. Let $\sigma<-1 / 2$. For $S B P$ operators of finite difference type, the matrix $\mathcal{D}=P^{-1}\left(Q-\mathbf{e}_{0} \mathbf{e}_{0}^{T}\right)$ has eigenvalues with strictly positive real parts if, and only if, $P^{-1} S$ does not have imaginary eigenvalues with eigenvectors where the first and last components are equal to zero.

To prove Assumption 5.0.1, we use the repeating stencil structure of $P^{-1} S$ to explicitly construct the interior components of the eigenvectors $\mathbf{v}_{1}, \mathbf{v}_{2}$ fulfilling $\left(\mathbf{v}_{1}\right)_{0}=0$ and $\left(\mathbf{v}_{2}\right)_{N}=0$, respectively. If the two vectors are linearly independent, an eigenvector $\mathbf{v}$ satisfying both $v_{0}=0$ and $v_{N}=0$ can not exist and the eigenvalue assumption is verified due to Lemma 5.2.1.

As an example, for the second order approximation we get

$$
P^{-1} S=\frac{1}{\Delta x}\left[\begin{array}{cccccc}
0 & 1 & & & & \\
-\frac{1}{2} & 0 & \frac{1}{2} & & & \\
& -\frac{1}{2} & 0 & \frac{1}{2} & & \\
& & \ddots & \ddots & \ddots & \\
& & & -\frac{1}{2} & 0 & \frac{1}{2} \\
& & & & -1 & 0
\end{array}\right]
$$

Note that this matrix does not depend on $\sigma$. Moreover, the mesh-size acts only as a rescaling parameter for the eigenvalues and the existence of eigenvectors $\mathbf{v}$ with $v_{0}=v_{N}=0$ does not depend on $\Delta x$. For this reason, we consider $\Delta x=1$ in (5.2).

On the other hand, while computing the eigenvectors of $P^{-1} S$ we can neither get rid of $N$ nor the order of accuracy. The parameter $N$ appears twice in the analysis, both as dimension of matrices and as explicit parameter in the computations. The computation being dependent on the order of accuracy implies that Assumption 5.0.1 must be verified independently for each order. In Paper III, we prove the eigenvalue assumption for second-, fourth- and sixthorder accurate finite-difference SBP operators.

\subsubsection{Proof for second-order accurate operators}

To illustrate our procedure, we sketch the proof for the second-order accurate SBP approximation.

Remark 5.2.1. For second order approximations it is trivial to verify that $P^{-1} S$ does not have eigenvectors with first and last components equal to zero [33]. We consider this case only to clearly explain the proof procedure used in Paper III for higher orders.

The eigenvalue problem $P^{-1} S \mathbf{v}=i \xi \mathbf{v}$ for the matrix in (5.2) gives rise to the internal stencil relation

$$
\frac{1}{2} v_{k+1}-\frac{1}{2} v_{k-1}=i \xi v_{k}, \quad k=1, \ldots, N-1 .
$$


This recurrence relation is solved by

$$
v_{k}=c_{1}(\xi) r_{1}^{k}(\xi)+c_{2}(\xi) r_{2}^{k}(\xi), \quad k=1, \ldots, N-1,
$$

with $r_{1,2}(\xi)=i \xi \pm \sqrt{1-\xi^{2}}$. The coefficients $c_{1,2}(\xi)$ can be found by solving explicitly for $v_{1}$ and $v_{2}$ the first two equations of the eigenvalue problem with $v_{0}$ as free parameter. In particular, by matching the components $v_{1}=i \xi v_{0}$, $v_{2}=\left(1-2 \xi^{2}\right) v_{0}$ with the solution in (5.3), we find the homogeneous linear rectangular system

$$
\left[\begin{array}{ccc}
r_{1} & r_{2} & -i \xi \\
r_{1}^{2} & r_{2}^{2} & 2 \xi^{2}-1
\end{array}\right]\left[\begin{array}{c}
c_{1}(\xi) \\
c_{2}(\xi) \\
v_{0}
\end{array}\right]=\left[\begin{array}{l}
0 \\
0
\end{array}\right]
$$

Solving for $c_{1,2}(\xi)$ with $v_{0}$ as free parameter yields $c_{1}^{L}(\xi)=c_{2}^{L}(\xi)=v_{0} / 2$.

Likewise, the coefficients in (5.3) can be found also by solving for $v_{N-2}$ and $v_{N-1}$ the last two equations of the eigenvalue problem with $v_{N}$ as free parameter:

$$
c_{1}^{R}(\xi)=-\frac{i \xi+r_{2}\left(1-2 \xi^{2}\right)}{r_{1}^{N-2}\left(r_{1}-r_{2}\right)} v_{N}, \quad c_{2}^{R}(\xi)=\frac{i \xi+r_{1}\left(1-2 \xi^{2}\right)}{r_{2}^{N-2}\left(r_{1}-r_{2}\right)} v_{N} .
$$

The existence of the eigenvectors of $P^{-1} S$ in (5.2) is subject to the linear dependence of $\mathbf{c}^{L}(\xi)=\left[c_{1}^{L}(\xi), c_{2}^{L}(\xi)\right]^{T}$ and $\mathbf{c}^{R}(\xi)=\left[c_{1}^{R}(\xi), c_{2}^{R}(\xi)\right]^{T}$. Vice versa, the linear independence of the coefficients can be used to exclude the existence of eigenvectors with $v_{0}=v_{N}=0$ for higher order approximations, thus verifying Assumption 5.0.1. This principle can be used in general to check the eigenvalue assumption for the approximations with a repeating stencil.

Remark 5.2.2. For second order approximations, $v_{0}=0$ and $v_{N}=0$ lead to $\mathbf{c}^{L}(\xi)=\mathbf{0}$ and $\mathbf{c}^{R}(\xi)=\mathbf{0}$, respectively. Hence, Assumption 5.0.1 holds in this case. 



\section{Dual time-stepping}

The Dual Time-Stepping (DTS) technique consists in adding a pseudo timederivative of the solution with respect to the so-called dual time and marching in that dual time to steady-state. This procedure can be used for solving large systems of nonlinear equations [20,3]. In Paper IV, we introduce a modified DTS formulation which makes use of two derivatives in pseudo-time. This new technique reduces the stiffness of the problem and improves the convergence rate to the stationary solution.

In this section, we briefly present and compare the classical and new DTS techniques. All the spatial approximations are performed with sixth-order centered finite-difference SBP operators, augmented with SAT terms weakly imposing the boundary conditions.

\subsection{The classical dual time-stepping}

Consider the one-dimensional advection equation

$$
u_{t}+a u_{x}=0, \quad x \in \Omega, \quad t>0,
$$

where $a$ is a positive constant and $\Omega$ the spatial domain. By discretizing the first derivative in space with the SBP operator $D$ and applying the Euler-backward scheme in time, we get

$$
\frac{\mathbf{u}^{n+1}-\mathbf{u}^{n}}{\Delta t}+a D \mathbf{u}^{n+1}=0
$$

Here, $\mathbf{u}^{n+1}$ and $\mathbf{u}^{n}$ represent the approximate solution at $t^{n+1}=(n+1) \Delta t$ and $t^{n}=n \Delta t$, respectively. To avoid the direct computation of $\mathbf{u}^{n+1}$, which may be expensive, we apply the DTS technique as

$$
\mathbf{w}_{\tau}+\frac{\mathbf{w}-\mathbf{u}^{n}}{\Delta t}+a D \mathbf{w}=0, \quad \tau>0 .
$$

This formulation, can be rewritten in compact form as

$$
\mathbf{w}_{\tau}+F \mathbf{w}=\mathbf{R}, \quad \tau>0,
$$

where $F=I / \Delta t+a D$ and $\mathbf{R}=\mathbf{u}^{n} / \Delta t$. If the solution $\mathbf{w}$ in (6.2) reaches steady-state, it will converge to $\mathbf{u}^{n+1}$ in (6.1). 
The same procedure can be applied to a general time-space discretization of a differential problem $F(\mathbf{u})=\mathbf{R}$. In Paper IV we assumed for simplicity that this problem is linear. If, additionally, $F$ is invertible and diagonalizable it is possible to determine when the DTS procedure (6.2) converges. We proved

Proposition 6.1.1. If the eigenvalues of $F$ have positive real parts, then the dual time-stepping procedure (6.2) converges to the steady-state solution $\mathbf{u}=$ $F^{-1} \mathbf{R}$.

Remark 6.1.1. The eigenvalue of $F$ with the minimum real part determines the convergence rate in (6.2).

Remark 6.1.2. The results of Paper II and III imply that DTS converges when applied to pseudo-spectral and finite-difference SBP-SAT time approximations of energy-stable spatial discretizations.

\subsection{The second-derivative DTS technique}

To possibly get a faster decay to steady-state, we considered the modified DTS formulation

$$
\mathbf{w}_{\tau \tau}+2 G \mathbf{w}_{\tau}+F \mathbf{w}=\mathbf{R}, \quad \tau>0 .
$$

In Paper IV, we analyzed this DTS technique assuming that the matrix $G$ has the same eigenvectors as $F$. We proved that the procedure with the fastest decay can be obtained by setting $G=F^{\frac{1}{2}}$, i.e. the principal square root of the matrix $F$ having eigenvalues with non-negative real parts.

The second-derivative DTS technique can hence be written as

$$
\mathbf{w}_{\tau \tau}+2 F^{\frac{1}{2}} \mathbf{w}_{\tau}+F \mathbf{w}=\mathbf{R}, \quad \tau>0 .
$$

This formulation generalizes the critically damped harmonic oscillator and converges to steady-state with optimal rate. In particular, we proved

Proposition 6.2.1. If the eigenvalues of $F$ do not lie on the negative real axis, then the new dual time-stepping procedure (6.4) converges to the steady-state solution $\mathbf{u}=F^{-1} \mathbf{R}$.

By comparing Proposition 6.1.1 and 6.2.1, we observe that the new DTS technique can drive the solution to steady-state when the classical one fails to do that. Furthermore, we proved that

Proposition 6.2.2. The decay to steady-state for the new DTS formulation (6.4) is determined by the principal square root of the eigenvalues of $F$. In particular, the square root with the minimum real part determines the convergence rate.

As a consequence of Proposition 6.2.2, the new DTS technique has a faster convergence to steady-state and reduces the stiffness of the problem. Indeed, 
Figure 6.1 indicates that the square root transforms complex numbers close to the imaginary axis into complex numbers with more positive real parts. It can be also observed that, when applied to complex numbers with large magnitude, the square root returns numbers less distant from the origin, thereby reducing the stiffness of the problem and allowing for larger dual time-steps.
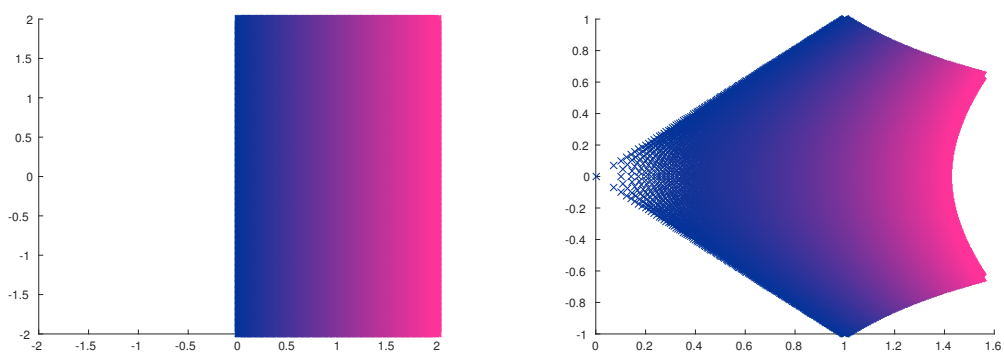

Figure 6.1: Complex numbers with non-negative real parts (left figure) and their square root. The distribution of points near the origin of the complex plane tends to rarefy. Furthermore, applying the square root to complex numbers with large magnitude yields numbers less distant from the origin.

\subsection{A numerical comparison}

Consider the following system

$$
\begin{aligned}
\mathbf{u}_{t}+A \mathbf{u}_{x} & =\varepsilon B \mathbf{u}_{x x}+F(x, t), & & 0<x<1, \quad t>0, \\
\mathbf{u}(x, 0) & =\mathbf{f}(x), & & 0<x<1, \\
\left(u_{1}+\sqrt{2} u_{2}-\varepsilon u_{2, x}\right)(0, t) & =g_{0}(t), & & t>0, \\
\left(u_{1}-\sqrt{2} u_{2}-\varepsilon u_{2, x}\right)(1, t) & =g_{1}(t), & & t>0,
\end{aligned}
$$

modelling the time-dependent compressible Navier-Stokes equations. In (6.5), we used $\mathbf{u}(x, t)=\left[u_{1}(x, t), u_{2}(x, t)\right]^{T}, \varepsilon=10^{-2}$ and the matrices

$$
A=\left[\begin{array}{ll}
0 & 1 \\
1 & 0
\end{array}\right], \quad B=\left[\begin{array}{ll}
0 & 0 \\
0 & 1
\end{array}\right]
$$

For this problem, we considered a set of characteristic boundary conditions which made the problem well-posed and its SBP-SAT space approximation energy-stable.

In Figure 6.2, we compared the number of iterations to convergence for the two DTS techniques applied to a fully-discrete approximation of (6.5). This discrete problem is obtained by using SBP-SAT in space and the second-order Backward Difference Formula (BDF2) in time. We used a fourth-order Runge-Kutta scheme as pseudo time-integrator and the stopping criterion $\left\|\mathbf{w}^{n}-\mathbf{u}\right\|_{P}<10^{-6}$. 

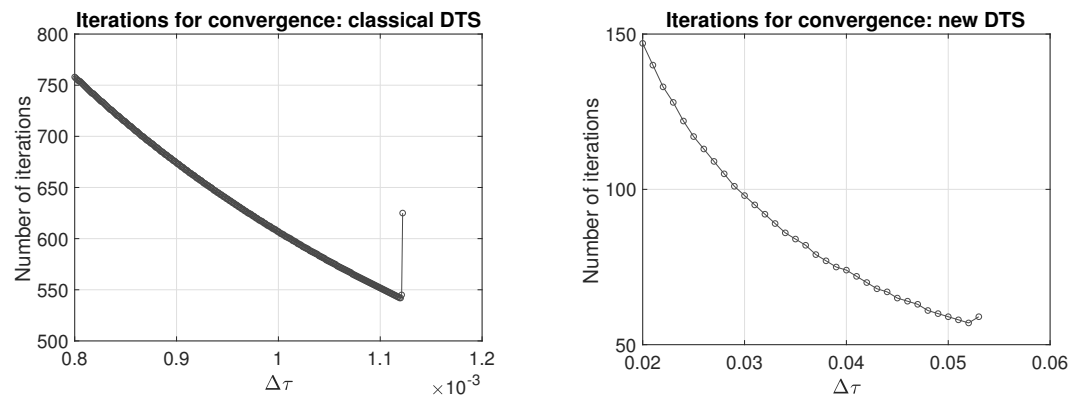

Figure 6.2: Number of iterations to convergence using the classical and the new DTS.

For $\Delta \tau=1.119 \cdot 10^{-3}$, the classical DTS technique reaches steady-state in 542 iterations while the new DTS technique converges in 57 inner iterations for the optimal dual time-step $\Delta \tau=0.052$. We conclude that, in this case, the new DTS required approximately ten times less iterations to convergence compared to the classical one.

\subsection{The main drawback and aftermath}

We showed that the new DTS technique (6.4) had better convergence properties compared to the conventional DTS (6.2). However, the computation of the principal square root of $F$ may be excessively expensive if the dimension of the system to solve is large, which is a major drawback. Nonetheless, Paper IV proves that it is theoretically possible to achieve faster convergence to steady-state by employing second derivatives in pseudo-time. Future research on this topic might reveal suboptimal formulations of (6.3) which do not involve fractional or negative powers of $F$, leading to a more efficient time-marching technique. Furthermore, less costly approximations of the matrix square root $F^{\frac{1}{2}}$ may shed new light on this approach. 


\section{Multi-grid techniques}

The Multi-Grid (MG) method is a convergence acceleration technique which complements iterative solvers with grid coarsening. As with the DTS, MG is often used to compute steady-state solutions for large systems of equations obtained from numerical approximations of IBVPs.

The MG method was originally designed for parabolic partial differential equations [11], for which iterative solvers effectively damp high frequency errors while maintaining smooth residuals unaltered after a number of iterations. However, the low frequency residuals can be efficiently reduced through grid coarsening, since these are interpreted as highly oscillating error modes on coarser grids $[42,30,15]$. MG methods for high-order finite-difference SBP-SAT approximations of parabolic problems are studied in Paper V.

Multi-grid methods were also developed for hyperbolic systems [31, 19]. For these problems, the error modes are expelled from the domain rather than damped: grid coarsening can be used to accelerate the convergence to steadystate, since coarser grids have milder restrictions on time steps. As an example, in [43] the authors present a $L$-level MG algorithm to find first order accurate steady-state solutions of hyperbolic conservation laws. This procedure allows for $2^{L}$ times faster wave propagation by ensuring that the Total Variation Diminishing (TVD) property is preserved. In Paper VI, the resulting TVD Multi-Grid (TVD-MG) scheme is reformulated by combining first order SBP upwind operators on coarse meshes with higher order operators on the fine mesh.

Consider the following IBVP

$$
\begin{aligned}
u_{t}+L u & =f, & & \text { in } \Omega, & t>0 \\
u & =u^{0}, & & \text { in } \Omega, & t=0, \\
H u & =g, & & \text { on } \partial \Omega, & t>0
\end{aligned}
$$

where $L$ and $H$ are a differential and boundary operator, respectively. Furthermore, $f$ and $g$ are given functions and $\Omega$ is the domain with boundary $\partial \Omega$. In the remainder of this section, we present the MG algorithms used in Paper V and VI to find an approximation to the steady-state solution of (7.1). 


\subsection{Multi-grid for parabolic problems}

To start with, we present MG algorithms for parabolic problems such as

$$
\begin{aligned}
u_{t}-u_{x x} & =f(x), \quad \alpha<x<\beta, \quad t>0, \\
u(x, 0) & =u^{0}(x), \quad \alpha<x<\beta
\end{aligned}
$$

with $f$ and $u^{0}$ known data independent of time. We also consider a suitable set of boundary conditions leading to the well-posedness of (7.2).

Consider a grid $\Omega_{1}=\left\{x_{j}^{(1)}=\alpha+j \Delta x^{(1)}, j=0,1, \ldots, N\right\} \subset \Omega=[\alpha, \beta]$, henceforth called the fine grid, and the following spatial approximation to (7.1)

$$
\begin{aligned}
\mathbf{u}_{t}+L_{1} \mathbf{u} & =\mathbf{F}_{1}, \quad \text { in } \Omega_{1}, \quad t>0 \\
\mathbf{u} & =\mathbf{U}^{0}, \quad \text { in } \Omega_{1}, \quad t=0 .
\end{aligned}
$$

In (7.3), $L_{1}$ is a discrete version of the operator $L$ in (7.1) augmented with the boundary conditions. The vector $\mathbf{F}_{1}$ is a grid function approximating $f$ on the nodes of $\Omega_{1}$, augmented with the boundary data $g$, while $\mathbf{u}$ is an approximate solution to $u$ in (7.1).

To compute the steady-state solution of (7.3), i.e. $\mathbf{U}=L_{1}^{-1} \mathbf{F}_{1}$, we use the following algorithm

Fine grid smoothing:

Fine grid residual:

Solution injection:

Coarse grid residual:

Coarse grid correction problem:

Fine grid update:

$$
\begin{aligned}
& \overline{\boldsymbol{U}}^{(1)}=S_{1} \boldsymbol{U}^{n}+\left(I_{1}-S_{1}\right) \boldsymbol{U} ; \\
& \overline{\boldsymbol{r}}^{(1)}=L_{1} \overline{\boldsymbol{U}}^{(1)}-\boldsymbol{F}_{1} ; \\
& \boldsymbol{U}^{(2)}=\mathcal{R}_{u} \overline{\boldsymbol{U}}^{(1)} ; \\
& \boldsymbol{F}_{2}=L_{2} \boldsymbol{U}^{(2)}-I_{r} \overline{\boldsymbol{r}}^{(1)} ; \\
& L_{2} \overline{\boldsymbol{U}}^{(2)}=\boldsymbol{F}_{2} ; \\
& \boldsymbol{U}^{n+1}=\overline{\boldsymbol{U}}^{(1)}+I_{p}\left(\overline{\boldsymbol{U}}^{(2)}-\mathcal{R}_{u} \overline{\boldsymbol{U}}^{(1)}\right) .
\end{aligned}
$$

Remark 7.1.1. The $M G$ algorithm (7.4) is equivalent to the one presented in Paper V. Here, it has been reformulated using the notation in Paper VI to ease the comparison with the procedure used for hyperbolic problems.

In (7.4), $S_{1}$ is an operator representing a few steps of an iterative method such as the third order Runge-Kutta (RK3), Weighted Jacobi (WJ) or the Successive Over Relaxation Method (SOR). Moreover, we have introduced an injection operator $\mathcal{R}_{u}$ which projects fine grid functions onto a coarse grid

$$
\Omega_{2}=\left\{x_{j}^{(2)}=\alpha+2 j \Delta x^{(1)}=\alpha+j \Delta x^{(2)}, j=0,2, \ldots, N\right\} .
$$

On this grid, we exactly solve the problem $L_{2} \overline{\boldsymbol{U}}^{(2)}=\boldsymbol{F}_{2}$, where $L_{2}$ is a counterpart of $L_{1}$ on $\Omega_{2}$. Finally, (7.4) makes use of a residual restriction $I_{r}$ and a prolongation operator $I_{p}$ which convey the information between $\Omega_{1}$ and $\Omega_{2}$. 
All these multi-grid ingredients play an important role for the convergence of the algorithm. In order to have a well-functioning procedure we require that the original steady-state problem $L_{1} \mathbf{U}=\mathbf{F}_{1}$ is compatible with the coarse-grid problem in (7.4). In Paper VI, we show that this compatibility constraint can be satisfied by the approximation

$$
I_{r} L_{1} \approx L_{2} \mathcal{R}_{u}
$$

\subsubsection{The Galerkin condition}

The multi-grid algorithm for parabolic problems (7.4) provides the following iterative method

$$
\mathbf{U}^{(n+1)}=M \mathbf{U}^{(n)}+\left(I_{1}-M\right) \mathbf{U},
$$

where $M=C S_{1}$ is called the multi-grid iteration matrix and $C=I_{1}-I_{p} L_{2}^{-1} I_{r} L_{1}$ is referred to as the coarse-grid correction operator. The form of the matrix $M$ shows that the two main components of the MG method, i.e. grid coarsening and smoothing by means of an iterative solver, are associated with two different matrices.

Remark 7.1.2. The asymptotic convergence factor of the iterative method is given by the spectral radius $\rho(M)$ of the matrix $M$, i.e. the maximum among the absolute values of its eigenvalues. Convergence is achieved when $\rho(M)<1$.

In Paper V, the coarse-grid operator $L_{2}$ is obtained through the so called Galerkin condition [6]

$$
L_{2}=I_{r} L_{1} I_{p}
$$

which leads to an idempotent coarse-grid correction matrix $C$, i.e. $C^{k}=C$ for $k \in \mathbb{N}$. We showed that the idempotency of $C$ implies that this operator deals with smooth parts of the error. Since $S_{1}$ naturally damps the remaining high frequency modes when applied to parabolic problems, the Galerkin condition (7.6) is a suitable choice.

\subsubsection{SBP-preserving interpolation operators}

For finite-difference SBP-SAT spatial approximations of (7.1), we can associate two scalar products induced by the norms $P_{1}$ and $P_{2}$

$$
\left(\phi_{1}, \psi_{1}\right)_{P_{1}}=\phi_{1}^{T} P_{1} \psi_{1}, \quad\left(\phi_{2}, \psi_{2}\right)_{P_{2}}=\phi_{2}^{T} P_{2} \psi_{2}
$$

related to the the fine- and coarse-grid operators $L_{1}$ and $L_{2}$. By enforcing that these two products are equal for $\phi_{1}=I_{p} \phi_{2}$ and $\boldsymbol{\psi}_{2}=I_{r} \boldsymbol{\psi}_{1}$, we get two interpolation operators $I_{r}$ and $I_{p}$ which are adjoint to each other with respect to the SBP-based norms $P_{1}$ and $P_{2}$. This observation leads to the following definition of SBP-preserving (or inner product preserving) interpolation operators $[26,1]$. 
Definition 7.1.1. Let the grid functions $\mathbf{x}_{1}^{k}$ and $\mathbf{x}_{2}^{k}$ be the projections of the monomials $x^{k}$ onto equidistant 1-D grids corresponding to a fine and coarse grid, respectively. We say that $I_{r}$ and $I_{p}$ fulfilling $I_{r}=P_{2}^{-1} I_{p}^{T} P_{1}$ are $2 q$ th-order accurate SBP-preserving interpolation operators if $I_{r} \mathbf{x}_{1}^{k}-\mathbf{x}_{2}^{k}$ and $I_{p} \mathbf{x}_{2}^{k}-\mathbf{x}_{1}^{k}$ vanish for $k=0, \ldots, 2 q-1$ in the interior and for $k=0, \ldots, q-1$ at the boundaries.

The SBP-preserving interpolation is used in Paper V to accelerate the convergence to steady-state for parabolic problems such as (7.2). We prove that these restriction and prolongation operators, in combination with the Galerkin condition (7.6), lead to first- and second-derivative coarse grid operators with the summation-by-parts property. Furthermore, we show that the resulting SBP operators retain the order of accuracy of their fine grid counterparts at the interior nodes. Finally, we numerically study the convergence to steady-state of MG methods when using SBP-preserving interpolation operators. The tests are performed comparing the spectral radius of the resulting multi-grid iteration matrix with the one obtained by using linear prolongation and restriction operators such that $I_{r}=I_{p}^{T} / 2$. This analysis confirms that the SBP-preserving interpolation leads to a significant improvement of the convergence rates.

Remark 7.1.3. The SBP-preserving relation $I_{r}=P_{2}^{-1} I_{p}^{T} P_{1}$, in contrast to $I_{r}=I_{p}^{T} / 2$, yields a second order restriction operator which is consistent at the boundaries, see Figure 7.1.
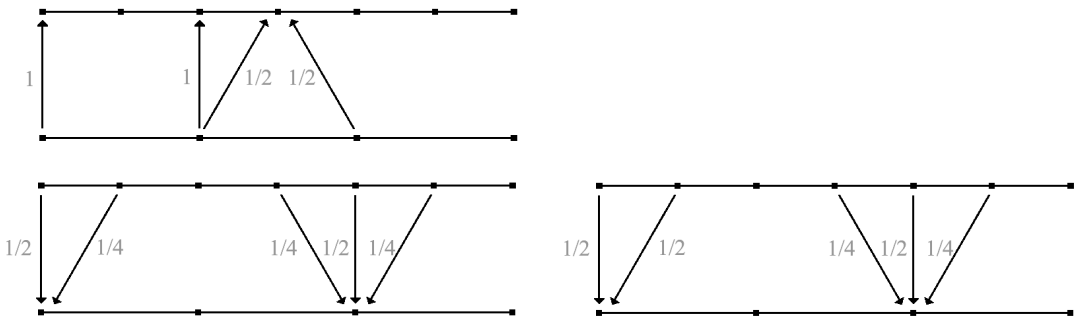

Figure 7.1: Linear prolongation $I_{p}$ (top left), conventional restriction $I_{r}=I_{p}^{T} / 2$ (bottom left) and second-order SBP-preserving restriction operator $I_{r}=P_{2}^{-1} I_{p}^{T} P_{1}$ (bottom right). The SBP-preserving relation leads to consistent restriction operators.

Remark 7.1.4. The second-derivative $S B P$ approximation $D_{2}=D^{2}$ does not modify modes with the Nyqust frequency. Since multi-grid methods for parabolic problems should damp high frequency error modes, for all numerical experiments in Paper V we use compact SBP operators (see section 3.1.1).

Remark 7.1.5. Because of the Galerkin condition, the approximation property (7.5) is satisfied only when $I_{p} \mathcal{R}_{u} \approx I_{1}$. Although this relation does not hold when 
using SBP-preserving prolongation operators, the $M G$ method (7.4) converges for all numerical experiments performed in Paper $V$.

\subsection{Multi-grid for hyperbolic problems}

Consider the linear wave propagation problem

$$
\begin{aligned}
u_{t}+u_{x} & =f(x), & & \alpha<x<\beta, \quad t>0, \\
u(0, t) & =g, & & t>0, \\
u(x, 0) & =u^{0}(x), & & \alpha<x<\beta,
\end{aligned}
$$

where $f, g$ and $u^{0}$ are known data independent of time.

As a first attempt to accelerate the convergence of the SBP-SAT spatial approximation of (7.7) to steady-state, we use the algorithm (7.4). In Figure 7.2, the logarithm of the spectral radius of the multigrid iteration matrix is shown as a function of the CFL number, i.e. $\Delta t / \Delta x$, for a second-order accurate discretization in space with centered finite-differences. For each multi-grid cycle, we used $\nu=5$ steps of RK3 as iterative solver. Although the SBP-preserving

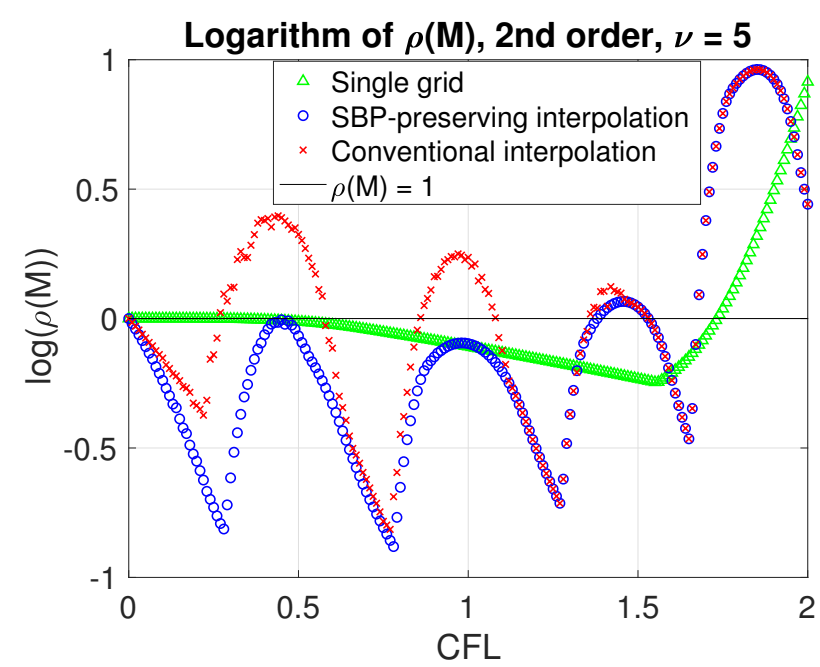

Figure 7.2: Logarithm of $\rho(M)$ for a second-order accurate SBP-SAT discretization of (7.7) with 101 equidistant grid points and $\nu=5$ steps of RK3.

interpolation leads to an improved MG procedure also for this problem, the CFL numbers that lead to convergence (i.e. for which $\rho(M)<1$ ) could not be predicted a priori. Hence, we concluded that the multi-grid algorithm (7.4) was not always convergent for the model problem (7.7). This phenomenon can 
be explained by the spurious oscillation occurring when using centered finitedifference stencils for hyperbolic problems. For this reason, wave propagation problems will be computed using SBP-SAT upwind spatial approximations.

\subsubsection{The TVD-MG algorithm}

A possible strategy to accelerate the convergence to steady-state of (7.7) is to use the TVD-MG algorithm proposed in [43]. This procedure was developed for first-order accurate spatial approximations and prevents spurious oscillations in the transient phase by preserving the TVD property. In Paper VI, we rewrite this algorithm in matrix-vector notation as

$$
\begin{aligned}
& \text { Fine grid propagation: } \quad \overline{\boldsymbol{U}}^{(1)}=S_{1} \boldsymbol{U}^{n}+\left(I_{1}-S_{1}\right) L_{1}^{-1} \boldsymbol{F}_{1} \text {; } \\
& \text { Fine grid residual: } \quad \overline{\boldsymbol{r}}^{(1)}=L_{1} \overline{\boldsymbol{U}}^{(1)}-\boldsymbol{F}_{1} \text {; } \\
& \text { Solution injection: } \quad \boldsymbol{U}^{(2)}=\mathcal{R}_{u} \overline{\boldsymbol{U}}^{(1)} \text {; } \\
& \text { Coarse grid residual: } \quad \boldsymbol{F}_{2}=L_{2} \boldsymbol{U}^{(2)}-I_{r} \overline{\boldsymbol{r}}^{(1)} \text {; } \\
& \text { Coarse grid propagation: } \quad \overline{\boldsymbol{U}}^{(2)}=S_{2} \boldsymbol{U}^{(2)}+\left(I_{2}-S_{2}\right) L_{2}^{-1} \boldsymbol{F}_{2} \text {; } \\
& \text { Fine grid update: } \quad \boldsymbol{U}^{n+1}=\overline{\boldsymbol{U}}^{(1)}+I_{p}^{I}\left(\overline{\boldsymbol{U}}^{(2)}-\mathcal{R}_{u} \overline{\boldsymbol{U}}^{(1)}\right) \\
& +I_{p}^{E}\left(\overline{\boldsymbol{U}}^{(2)}-\mathcal{R}_{u} \boldsymbol{U}^{n}\right) \text {. }
\end{aligned}
$$

In order to replicate the results given in [43], we introduce the new first-order SBP upwind operator in (3.5), which is compatible with the definition given in [25]. Numerical experiments show that this algorithm provides four times faster wave propagation, similar to the one used in [43].

Before we move on to the extension to higher order discretizations, we compare the MG algorithms (7.4) and (7.8). First of all, for hyperbolic problems we do not directly solve the coarse grid correction problem $L_{2} \overline{\boldsymbol{U}}^{(2)}=\boldsymbol{F}_{2}$ in (7.4): we rather use an iterative method based on time-marching to replicate the fine-grid wave propagation on the coarse grid. Secondly, in order to preserve the TVD property for first-order schemes, the fine grid update for (7.8) is performed by means of two prolongation operators, $I_{p}^{I}$ and $I_{p}^{E}$. The first of these two operators deals only with the grid nodes included on the coarse grid, while the second one considers the other nodes. In particular, $I_{p}^{I}=\mathcal{R}_{u}^{T}$ projects coarse grid functions onto the fine grid, see Figure 7.3.

The residual restriction operator $I_{r}$ used in (7.8) is said to be upwind-biased [43, 2 ], in contrast to the centered restriction operators used for parabolic problems (cf. Figure 7.1). In other words, wave propagation problems are accelerated with TVD-MG algorithms using residual restriction operators $I_{r}$ biased by the upwind direction. 

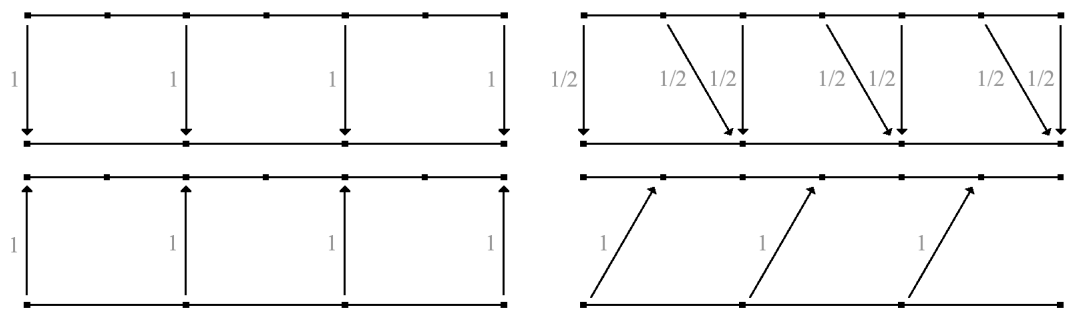

Figure 7.3: The interpolation operators used in the two-level algorithm (7.8) are the injection operator $\mathcal{R}_{u}$ (top left), the restriction operator $I_{r}$ (top right), the prolongation operator for the included nodes $I_{p}^{I}$ (bottom left) and the prolongation operator for the excluded nodes $I_{p}^{E}$ (bottom right). Note that $I_{r}$ is inconsistent at the left boundary node.

\subsubsection{Extension to higher order methods and systems}

The interpolation operator $I_{r}$ in Figure 7.3 is inconsistent at the left boundary node. However, this choice ensures that the approximation property (7.5) holds and indicates why the algorithm (7.8) leads to optimal convergence for first order schemes. To extend the MG algorithm to high order upwind SBP-SAT spatial approximations of (7.7), we consider residual restriction operators $I_{r}$ satisfying $I_{r} L_{1}=L_{2} \mathcal{R}_{u}$. If the fine grid operator $L_{1}$ is invertible, this interpolation operator is unique and given by $I_{r}=L_{2} \mathcal{R}_{u} L_{1}^{-1}$.

In this relation, $\mathcal{R}_{u}$ and $L_{1}$ are fixed, while $L_{2}$ depends on the order of accuracy chosen for the coarse grid correction problem. Since the order of convergence of MG does not depend on the accuracy of the operators on the coarse grids, a first order upwind scheme is used to construct $L_{2}$. This choice yields restriction operators $I_{r}$ which depend only on the order of accuracy of the fine grid discretization and the direction of the wave propagation. Furthermore, it gives rise to a straightforward generalization of algorithms involving more than two grids, since in this fashion the TVD-MG algorithm in [43] can be used with almost no modification for coarser grids.

In Paper VI, we show that the new $L$-grid algorithm for high order methods leads to $2^{L}$ times faster wave propagation, as with the TVD-MG for first order schemes. Moreover, we present an extension to hyperbolic systems which retains the same pleasant feature. As an example, in Figure 7.4 we show the convergence plots for the fifth- and sixth-order approximations of the linearized one dimensional symmetrized compressible Euler equations with characteristic boundary conditions. Analogous convergence plots are provided for a set of non-characteristic boundary conditions in Figure 7.5. In this case the leftand right-traveling waves are partly reflected back into the domain. This effect introduces dissipation and characterizes the convergence of the problem to 

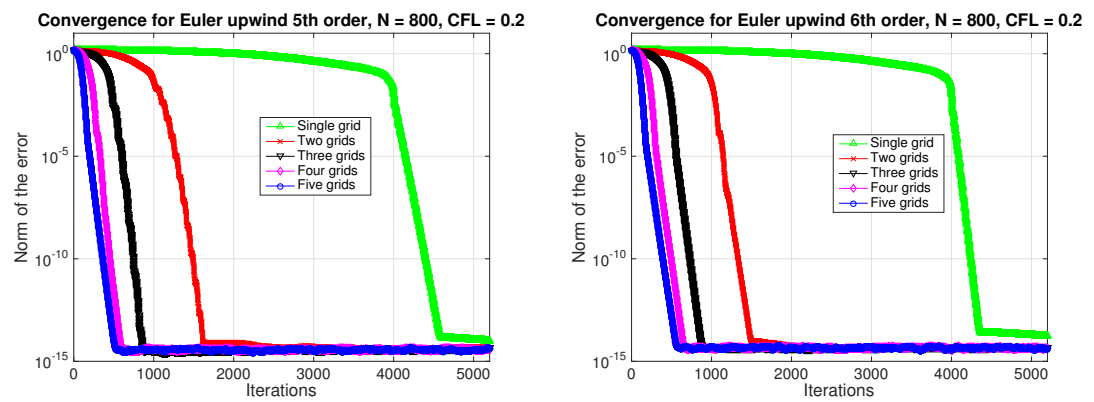

Figure 7.4: Convergence plots for the multigrid algorithm (7.8) applied to the fifth- and sixth-order approximations of the linearized one dimensional symmetrized form of the compressible Euler equations with characteristic boundary conditions.

steady-state. Nonetheless, the new MG algorithm accelerates the convergence by increasing the convergence rate proportionally to the number of grids used.
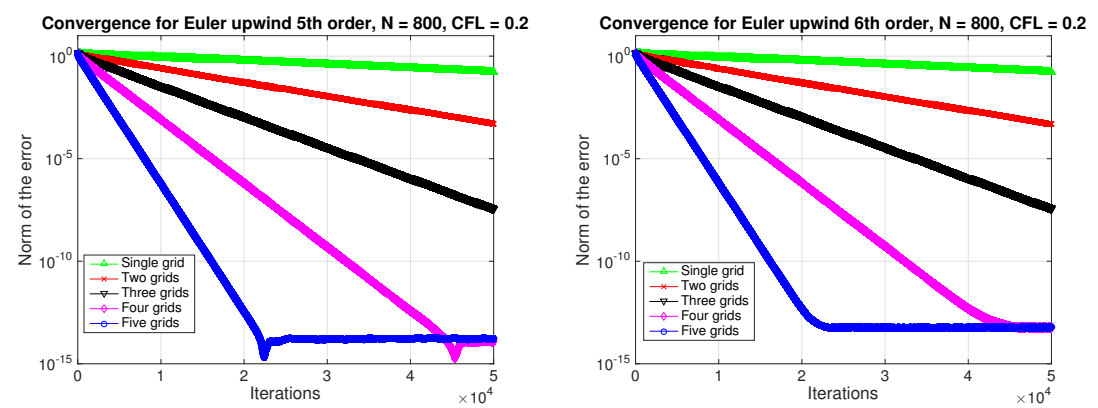

Figure 7.5: Convergence plots for the multigrid algorithm (7.8) applied to the fifth- and sixth-order approximations of the linearized one dimensional symmetrized form of the compressible Euler equations with a set of non-characteristic boundary conditions. 


\section{Summary of papers}

\section{Paper I: On conservation and stability properties for summation-by-parts schemes}

In Paper I, we show that conservative and stable numerical approximations of variable coefficient problems require a boundary and interface conforming grid. As a model problem, the linear advection equation with a variable coefficient is considered. We provide a conservative and stable single- and multi-block SBP-SAT approximation of this problem. It is also shown that GSBP-SAT formulations can be either conservative or stable.

\section{Paper II: On pseudo-spectral time discretizations in summation-by-parts form}

In Paper II, we prove that (G)SBP-SAT pseudo-spectral methods in time leads to discrete operators having eigenvalues with strictly positive real parts. This result also implies that pseudo-spectral collocation methods for the time derivative lead to invertible systems when energy-stable spatial approximations are used.

\section{Paper III: Eigenvalue analysis for summation-by- parts finite difference time discretizations}

Paper III deals with the same problem as in Paper II, but for finite-difference based time integration methods in summation-by-parts form. We prove that the second, fourth and sixth order time discretizations have eigenvalues with strictly positive real parts. Furthermore, we provide a general procedure to prove the same result for even higher order summation-by-parts approximations with repeating stencil.

\section{Paper IV: Dual time-stepping using second deriva- tives}

A new DTS technique with two derivatives in pseudo-time is studied and compared with the usual DTS, in Paper IV. The new formulation improves the 
decay rate compared to the classical time-marching if the eigenvalues of the operator $F$ representing the system are near the imaginary axis. Furthermore, the new second-derivative DTS reduces the stiffness of the problem if the spectrum resides outside the unitary circle. As a drawback, the computation of the principal square root of $F$ is required.

\section{Paper V: A new multigrid formulation for high order finite difference methods on summation-by- parts form}

In Paper V, the Galerkin condition is used in combination with the SBPpreserving interpolation operators to accelerate the convergence to steady-state of multi-grid methods applied to SBP-SAT finite-difference approximations of parabolic problems. The new procedure is compared with the conventional MG algorithm, which makes use of prolongation and restriction operators based on linear interpolation procedures, regardless of the accuracy of the discretization. Clear improvements of the convergence rate are achieved for the Poisson problem, anisotropic elliptic equations and advection diffusion problems, irrespective of the order of the discretization.

\section{Paper VI: Multigrid schemes for high order dis- cretizations of hyperbolic problems}

Paper VI deals with multi-grid methods for hyperbolic IBVPs. For these problems, we replicate the TVD-MG algorithm for first order schemes by using first order upwind SBP-SAT finite difference approximations. The MG algorithm for hyperbolic problems is then extended to high-order methods by complementing the wave propagation with the TVD-MG for first order schemes on coarse grids. The new $L$-grid algorithm for high order upwind SBP-SAT approximations leads to $2^{L}$ times faster wave propagation. 


\section{References}

[1] Oskar Ålund and Jan Nordström. Encapsulated high order difference operators on curvilinear non-conforming grids. Journal of Computational Physics, 385:209-224, 2019.

[2] Swathi Amarala and Justin W. L. Wan. Multigrid methods for systems of hyperbolic conservation laws. Multiscale modeling $\&$ simulations, 11:586$614,2013$.

[3] Andrey Belov, Luigi Martinelli, and Antony Jameson. A new implicit algorithm with multigrid for unsteady incompressible flow calculations. In 33rd AIAA Aerospace Sciences Meeting, AIAA paper 95-0049, Reno, NV, January 1995.

[4] Jens Berg and Jan Nordström. Superconvergent functional output for timedependent problems using finite differences on summation-by-parts form. Journal of Computational Physics, 231:6846-6860, 2012.

[5] Pieter D. Boom and David W. Zingg. High-order implicit time-marching methods based on generalized summation-by-parts operators. Journal of Scientific Computing, 37(6):A2682-A2709, 2015.

[6] William L. Briggs, Van Emden Henson, and Stephen F. McCormick. A Multigrid Tutorial, second edition. SIAM, 2000.

[7] David Carlson. Controllability, inertia, stability for tridiagonal matrices. Linear Algebra and its Applications, 56:207-220, 1984.

[8] David Carlson, Biswa N. Datta, and Charles R. Johnson. A semi-definite lyapunov theorem and the characterization of tridiagonal d-stable matrices. SIAM Journal on Matrix Analysis and Applications, 3:293-304, 1982.

[9] Mark H. Carpenter, Jan Nordström, and David Gottlieb. A stable and conservative interface treatment of arbitrary spatial accuracy. Journal of Computational Physics, 148(2):341-365, 1999.

[10] David C. Del Rey Fernández, Pieter D. Boom, and David W. Zingg. A generalized framework for nodal first derivative summation-by-parts operators. Journal of Computational Physics, 266:214-239, 2014.

[11] Radiy P. Fedorenko. Iterative methods for elliptic difference equations. Russian Mathematical Surveys, 28(2):129-195, 1973.

[12] Travis C. Fisher, Mark H. Carpenter, Jan Nordström, Nail K. Yamaleev, and Charles Swanson. Discretely conservative finite-difference formulations for nonlinear conservation laws in split form: Theory and boundary conditions. Journal of Computational Physics, 234:353-375, 2013. 
[13] Bertil Gustafsson. On the implementation of boundary conditions for the method of lines. BIT Numerical Mathematics, 38(2):293-314, 1998.

[14] Bertil Gustafsson, Heinz-Otto Kreiss, and Joseph Oliger. Time dependent problems and difference methods. John Wiley \& Sons Inc., 1995.

[15] Wolfgang Hackbusch. Multi-Grid Methods and Applications. Springer, 1985 .

[16] Jacques Hadamard. Sur le problèmes aux dérivées partielles et leur signification physique. Princeton University Bulletin, 13:49-52, 1902.

[17] Ernst Hairer and Gerhard Wanner. Solving Ordinary Differential Equations II: Stiff and Differential Algebraic Problems. Springer Series in Computational Mathematics, 2010.

[18] Jason E. Hicken and David W. Zingg. Dual consistency and functional accuracy: a finite-difference perspective. Journal of Computational Physics, 256:161-182, 2014.

[19] Antony Jameson. Solution of the Euler equations for two dimensional transonic flow by a multigrid method. Applied Mathematics and Computation, 13:327-355, 1983.

[20] Antony Jameson. Time dependent calculations using multigrid, with applications to unsteady flows past airfoils and wings. In 10th AIAA Computational Fluid Dynamics Conference, AIAA paper 91-1596, Honolulu, HI, June 1991.

[21] Heinz-Otto Kreiss and Godela Scherer. Finite element and finite difference methods for hyperbolic partial differential equations. In Mathematical Aspects of Finite Elements in Partial Differential Equations. Academic Press, 1974.

[22] V. Linders, J. Nordström, and Steven H. Frankel. Convergence and stability properties of summation-by-parts in time. Linköping University Press. Technical report, LiTH-MAT-R-2019/04-SE, 2019.

[23] Tomas Lundquist and Jan Nordström. The SBP-SAT technique for initial value problems. Journal of Computational Physics, 270:86-104, 2014.

[24] Ken Mattsson. Summation by parts operators for finite difference approximations of second-derivatives with variable coefficients. Journal of Scientific Computing, 51:650-682, 2012.

[25] Ken Mattsson. Diagonal-norm upwind sbp operators. Journal of Computational Physics, 335:283-310, 2017.

[26] Ken Mattsson and Mark H. Carpenter. Stable and accurate interpolation operators for high-order multi-block finite-difference methods. Journal of Scientific Computing, 32(4):2298-2320, 2010. 
[27] Ken Mattsson and Jan Nordström. Summation by parts operators for finite difference approximations of second derivatives. Journal of Computational Physics, 199(2):503-540, 2004.

[28] Ken Mattsson and Jan Nordström. High order finite difference methods for wave propagation in discontinuous media. Journal of Computational Physics, 220:249-269, 2006.

[29] Ken Mattsson, Magnus Svärd, and Jan Nordström. Stable and accurate artificial dissipation. Journal of Scientific Computing, 21(1):57-79, 2004.

[30] Stephen F. McCormick. Multigrid Methods. SIAM, 1987.

[31] Ron-ho Ni. A multiple-grid scheme for solving the Euler equations. AIAA Journal, 20:1565-1571, 1982.

[32] Jan Nordström. Conservative finite difference formulations, variable coefficients, energy estimates and artificial dissipation. Journal of Scientific Computing, 29(3):375-404, 2006.

[33] Jan Nordström and Tomas Lundquist. Summation-by-parts in time. Journal of Computational Physics, 251:487-499, 2013.

[34] Pelle Olsson. Summation by parts, projections, and stability. I. Mathematics of Computation, 64(211):1035-1065, 1995.

[35] N. Anders Petersson, Ossian O'Reilly, Björn Sjögreen, and Samuel Bydlon. Discretizing singular point sources in hyperbolic wave propagation problems. Journal of Computational Physics, 321:532-555, 2016.

[36] Hendrik Ranocha. Generalised summation-by-parts operators and variable coefficients. Journal of Computational Physics, 362:20-48, 2018.

[37] Hendrik Ranocha. Some notes on summation by parts time integration methods. Technical report, Technische Universität Braunschweig, Institute of Computational Mathematics, 2019.

[38] Hendrik Ranocha, Philipp Öffner, and Thomas Sonar. Summation-by-parts operators for correction procedure via reconstruction. Journal of Computational Physics, 311:299-328, 2016.

[39] Bo Strand. Summation by parts for finite difference approximations for d/dx. Journal of Computational Physics, 110:47-67, 1994.

[40] Magnus Svärd. On coordinate transformation for summation-by-parts operators. Journal of Scientific Computing, 110:29-42, 2004.

[41] Magnus Svärd and Jan Nordström. Review of summation-by-parts schemes for initial-boundary-value problems. Journal of Computational Physics, 268:17-38, 2014. 
[42] Ulrich Trottenberg, Cornelius W. Oosterlee, and Anton Schuller. Multigrid. Academic Press, 2000.

[43] Justin W. L. Wan and Antony Jameson. Monotonicity preserving multigrid time stepping schemes for conservation laws. Computing and Visualization in Science, 11:41-58, 2008. 


\section{Papers}

The papers associated with this thesis have been removed for copyright reasons. For more details about these see:

http://urn.kb.se/resolve?urn=urn:nbn:se:liu:diva-160073 


\section{FACULTY OF SCIENCE AND ENGINEERING}

Linköping Studies in Science and Technology, Dissertation No. 2002, 2019

Department of Mathematics

Linköping University

SE-581 83 Linköping, Sweden

www.liu.se 